\title{
Room Temperature Catalyst System for the Hydroarylation of Olefins
}

\author{
Siu Yin Lee, Alexander J. Villani-Gale, and Chad C. Eichman* \\ Department of Chemistry and Biochemistry, Loyola University Chicago \\ 1068 West Sheridan Road, Chicago, Illinois 60660, United States
}

Table of Contents

Table of Contents

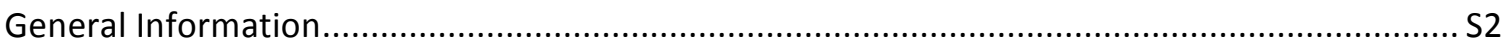

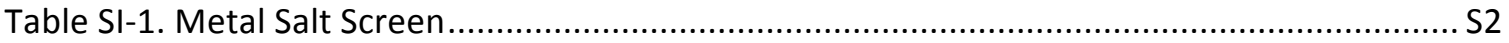

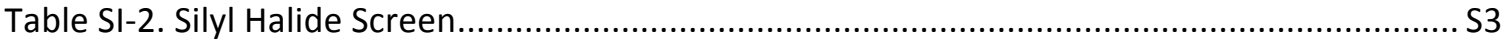

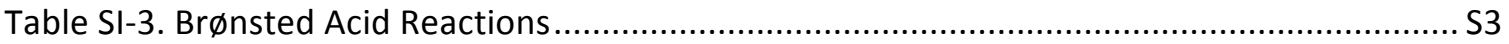

General Procedure for the Hydroarylation of Styrene ............................................................ 44

Procedure for the Hydroarylation of Styrene with 1-Methylindole ............................................ S7

General Procedure for the Hydroarylation of Alkyl Olefins with Anisole....................................S9

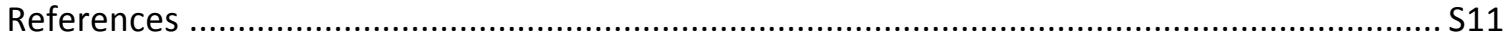

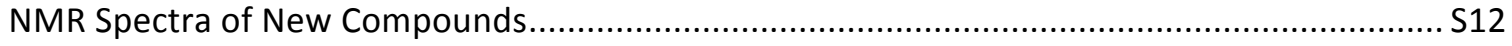

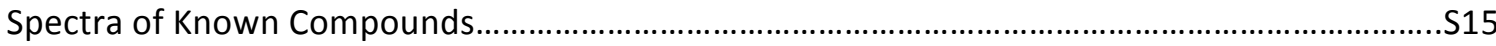




\section{General Information}

All hydroarylation reactions were conducted in oven- or flame-dried glassware with magnetic stirring. 1,2Dichloroethane (DCE), tetrahydrofuran (THF) and anisole were distilled prior to use. Styrene was purified by passing through an alumina column. Other reagents were used as purchased unless otherwise noted. Analytical thin layer chromatography (TLC) was performed on Merck Silica Gel 60-F plates and visualized with UV light. Flash column chromatography was carried out using silica gel 60 (230-400 mesh). ${ }^{1} \mathrm{H}$ nuclear magnetic resonance (NMR) spectra were recorded on a Varian INOVA $500 \mathrm{MHz}$ spectrometer. Chemical shifts are reported in ppm using tetramethylsilane (TMS) as an internal standard (0 ppm). Data are reported as: $\mathrm{s}=$ singlet, $\mathrm{d}=$ doublet, $\mathrm{t}=$ triplet, $\mathrm{q}=$ quartet, $\mathrm{sep}=$ septet, $\mathrm{m}=$ multiplet, $\mathrm{b}=$ broad. Coupling constant(s) are measured in $\mathrm{Hz}$. Decoupled ${ }^{13} \mathrm{C} N \mathrm{NMR}$ spectra were recorded at $75 \mathrm{MHz}$ on Varian INOVA $300 \mathrm{MHz}$ spectrometer with $\mathrm{CDCl}_{3}$ as the internal standard (77.15 ppm). GC-MS spectra were obtained on Agilent 6890N with a Hewlett-Packard 5973 quadrupole mass spectrometer (EI). High Resolution Mass Spectrometry were recorded by the Mass Spectrometry \& Proteomics Facility at the University of Notre Dame on a Bruker micrOTOF II.

\section{Table SI-1. Metal Salt Screen}<smiles>COc1ccc(C(C)c2ccccc2C(C)(C)c2ccccc2)cc1</smiles>

\begin{tabular}{|c|c|c|c|c|}
\hline \multirow{2}{*}{ Entry } & \multirow{2}{*}{ Metal Salt } & \multicolumn{2}{|c|}{ Yield (\%) ${ }^{a}$} & \multirow{2}{*}{$\begin{array}{l}\text { Selectivity } \\
(p-/ o-)^{a}\end{array}$} \\
\hline & & $1 a$ & $1 \mathrm{~b}$ & \\
\hline 1 & $\mathrm{AgSbF}_{6}$ & 68 & 16 & $4: 1$ \\
\hline 2 & $\mathrm{AgBF}_{4}$ & 65 & 15 & $4: 1$ \\
\hline 3 & $\mathrm{AgPF}_{6}$ & 68 & 18 & $4: 1$ \\
\hline 4 & $\mathrm{CoCl}_{2}$ & 46 & 8 & $6: 1$ \\
\hline 5 & $\mathrm{SnCl}_{2}$ & 65 & 13 & $5: 1$ \\
\hline 6 & $\mathrm{FeBr}_{2}$ & 61 & 13 & $5: 1$ \\
\hline 7 & $\mathrm{MnCl}_{2}$ & 51 & 15 & $3: 1$ \\
\hline 8 & $\mathrm{TiCl}_{4}$ & 62 & 19 & $3: 1$ \\
\hline 9 & $\mathrm{AlCl}_{3}$ & 62 & 11 & $6: 1$ \\
\hline
\end{tabular}

Note: A solvent screen revealed that 1,2-DCE provided the highest conversion of starting reagents. Screened solvents included PhMe, DCM, hexanes, 2-MeTHF. Benzene, and THF. 
Table SI-2. Silyl Halide Screen<smiles>COc1ccc(C(C)c2ccccc2)cc1</smiles>

\begin{tabular}{|c|c|c|c|c|}
\hline \multirow[b]{2}{*}{ Entry } & \multirow{2}{*}{$\begin{array}{l}\text { Halosilane } \\
(\mathrm{mol} \%)\end{array}$} & \multirow{2}{*}{$\begin{array}{l}\mathrm{ZnBr}_{2} \quad(\mathrm{~mol} \\
\%)\end{array}$} & \multicolumn{2}{|c|}{ Yield (\%) $)^{a}$} \\
\hline & & & $1 \mathrm{a}$ & $1 \mathrm{~b}$ \\
\hline 1 & TIPSCl (10) & 2 & 75 & 17 \\
\hline 2 & $\operatorname{DMSCl}(10)$ & 2 & 75 & 17 \\
\hline 3 & TPSCl (10) & 2 & 67 & 15 \\
\hline 4 & TMSI (10) & 2 & 68 & 15 \\
\hline
\end{tabular}

a Yield was determined by GC/MS analysis of the crude reaction mixture. TMSCI = chlorotrimethylsilane; $\quad \mathrm{TIPSCl}=$ chlorotriisopropylsilane; $\mathrm{DMSCl}=$ chlorodimethylsilane; TPSCI: chlorotriphenylsilane; TMSI: iodotrimethylsilane.

\section{Table SI-3. Brønsted Acid Reactions}

Acid Source (mol \%)
$\mathrm{ZnBr}_{2}(\mathrm{~mol} \mathrm{\%})$




\section{General Procedure for the Hydroarylation of Styrene}

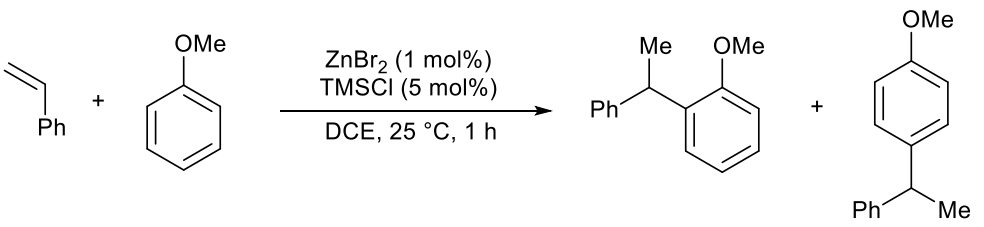

1-Methoxy-2-(1-phenylethyl)benzene (1a) and 1-methoxy-4-(1-phenylethyl)benzene (1b) (mixture of approximately $1: 4$ ortho and para isomers): To a $4 \mathrm{~mL}$ screw-capped vial with $\mathrm{ZnBr}_{2}\left(2 \mathrm{mg}, 8.9 \times 10^{-3} \mathrm{mmol}\right)$ open to air, 1,2-dichloroethane $(800 \mu \mathrm{L})$ and chlorotrimethylsilane $(6 \mu \mathrm{L}, 0.047 \mathrm{mmol})$ were added. Anisole (95 $\mu \mathrm{L}, 0.874 \mathrm{mmol})$ was then added followed by styrene $(100 \mu \mathrm{L}, 0.872 \mathrm{mmol})$. The reaction mixture was stirred at room temperature for 1 hour. A $10 \mu \mathrm{L}$ aliquot of this solution was removed for GC-MS analysis. The rest of the solution was filtered through a pad of celite $(1 \mathrm{~cm}, \mathrm{DCM}$ wash) and the solvent was evaporated to dryness. The crude reaction mixture was purified by column chromatography (hexane:EtOAc $=9: 1$ ) to obtain the $159 \mathrm{mg}$ ( $86 \%$ combined yield) of a colorless oil. The analytical data for this compound matched with the previously reported compound ${ }^{1}$ : ${ }^{1} \mathbf{H}$ NMR (500 $\left.\mathbf{M H z}^{\mathbf{C}} \mathbf{C D C l}_{3}\right)$ : $\delta$ (major isomer, $\left.1 \mathrm{a}\right) 7.32-7.10(\mathrm{~m}, 7 \mathrm{H}), 6.84-$ $6.82(\mathrm{~m}, 2 \mathrm{H}), 4.14(\mathrm{q}, J=7.5 \mathrm{~Hz}, 1 \mathrm{H}), 3.80(\mathrm{~s}, 3 \mathrm{H}), 1.64(\mathrm{~d}, J=7.5 \mathrm{~Hz}, 3 \mathrm{H})$; (minor isomer, 1b) $7.32-7.00(\mathrm{~m}$, $7 \mathrm{H}), 6.87-6.84(\mathrm{~m}, 2 \mathrm{H}), 4.62(\mathrm{q}, J=7.5 \mathrm{~Hz}, 1 \mathrm{H}), 3.80(\mathrm{~s}, 3 \mathrm{H}), 1.61(\mathrm{~d}, J=7.5 \mathrm{~Hz}, 3 \mathrm{H})$; LRMS (ESI): $\mathrm{m} / \mathrm{z} \mathrm{calcd}$ for both isomers for $\mathrm{C}_{15} \mathrm{H}_{16} \mathrm{O}[\mathrm{M}]^{+}$: 212.1; found: 212.1<smiles>Cc1cccc(C(C)(C)c2ccccc2)c1O</smiles>
and<smiles>Cc1cc(C(C)c2ccccc2)ccc1O</smiles>

2-Methyl-6-(1-phenylethyl)phenol (2a) and 2-methyl-4-(1-phenylethyl)phenol (2b) (mixture of approximately $4: 1$ ortho and para isomers): Following the general procedures using 0 -cresol (95 mg, 0.878 $\mathrm{mmol}$ ) and purified by column chromatography to afford $141 \mathrm{mg}$ ( $76 \%$ combined yield) of a colorless oil. The analytical data for this compound matched with the previously reported compound ${ }^{2}$ : ${ }^{\mathbf{1}} \mathbf{H} \mathbf{~ N M R}(\mathbf{5 0 0} \mathbf{~ M H z}$; $\left.\mathrm{CDCl}_{3}\right): \delta$ (major isomer) $7.31-7.10(\mathrm{~m}, 6 \mathrm{H}), 6.73-6.66(\mathrm{~m}, 2 \mathrm{H}), 4.55(\mathrm{bs}, 1 \mathrm{H}), 4.31(\mathrm{q}, J=7.2 \mathrm{~Hz}), 2.19(\mathrm{~s}, 3$ H), $1.64(\mathrm{~d}, J=7.2 \mathrm{~Hz}, 3 \mathrm{H})$; (minor isomer) $7.31-7.10(\mathrm{~m}, 6 \mathrm{H}) 6.83-6.80(\mathrm{~m}, 2 \mathrm{H}), 4.50(\mathrm{bs}, 1 \mathrm{H}), 4.04(\mathrm{q}, J=$ $7.2 \mathrm{~Hz}, 1 \mathrm{H}), 2.20(\mathrm{~s}, 3 \mathrm{H}), 1.58(\mathrm{~d}, J=7.2 \mathrm{~Hz}, 1 \mathrm{H})$; LRMS (ESI): m/z calcd for both isomers for $\mathrm{C}_{15} \mathrm{H}_{16} \mathrm{O}\left[\mathrm{M}^{+}\right.$: 212.1; found: 212.1

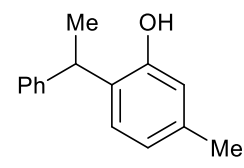

5-Methyl-2-(1-phenylethyl)phenol (3): Following the general procedures using $\mathrm{m}$-cresol (95 $\mathrm{mg}, 0.878 \mathrm{mmol}$ ) and purified by column chromatography to afford $124 \mathrm{mg}$ (67\% yield) of a colorless oil. The analytical data for this compound matched with the previously reported compound ${ }^{2}:{ }^{1} \mathbf{H} \mathbf{N M R}\left(\mathbf{5 0 0} \mathbf{~ M H z} ; \mathbf{C D C l}_{3}\right): \delta 7.28$ - 
$7.12(\mathrm{~m}, 6 \mathrm{H}), 6.68-6.62(\mathrm{~m}, 2 \mathrm{H}), 4.50(\mathrm{bs}, 1 \mathrm{H}), 4.24$ (q, , J = 7.2 Hz, $1 \mathrm{H}), 2.17(\mathrm{~s}, 3 \mathrm{H}), 1.57(\mathrm{~d}, J=7.2 \mathrm{~Hz}, 3 \mathrm{H})$ ; LRMS (ESI): $\mathrm{m} / \mathrm{z}$ calcd for $\mathrm{C}_{15} \mathrm{H}_{16} \mathrm{O}[\mathrm{M}]^{+}:$:212.1; found: 212.1<smiles>Cc1ccc(O)c(C(C)c2ccccc2)c1</smiles>

4-Methyl-2-(1-phenylethyl)phenol (4): Following the general procedures using $p$-cresol (95 $\mathrm{mg}, 0.878 \mathrm{mmol}$ ) and purified by column chromatography to afford $127 \mathrm{mg}$ (69\% yield) of a colorless oil. The analytical data for this compound matched with the previously reported compound ${ }^{2}:{ }^{1} \mathbf{H} \mathbf{N M R}\left(\mathbf{5 0 0} \mathbf{~ M H z} \mathbf{C D C l}_{3}\right): \delta 7.29-$ $7.13(\mathrm{~m}, 5 \mathrm{H}), 7.03(\mathrm{~d}, J=7.2 \mathrm{~Hz}, 1 \mathrm{H}), 6.91(\mathrm{~d}, J=7.8 \mathrm{~Hz}, 1 \mathrm{H}) 6.65(\mathrm{~d}, J=7.8 \mathrm{~Hz}, 1 \mathrm{H}), 4.42(\mathrm{bs}, 1 \mathrm{H}), 4.33(\mathrm{q}, J$ $=7.2 \mathrm{~Hz}, 1 \mathrm{H}), 2.27(\mathrm{~s}, 3 \mathrm{H}), 1.59(\mathrm{~d}, J=7.2 \mathrm{~Hz}, 3 \mathrm{H})$; LRMS (ESI): m/z calcd for $\mathrm{C}_{15} \mathrm{H}_{16} \mathrm{O}[\mathrm{M}]^{+}$: 212.1; found: 212.1<smiles>[M]C(c1ccccc1)c1cc(C)cc(C)c1O</smiles>

2,4-Dimethyl-6-(1-phenylethyl)phenol (5): Following the general procedures using 2,4-dimethylphenol (107 $\mathrm{mg}, 0.876 \mathrm{mmol}$ ) and purified by column chromatography to afford $158 \mathrm{mg}$ ( $80 \%$ yield) of a colorless oil. The analytical data for this compound matched with the previously reported compound ${ }^{3}$ : ${ }^{\mathbf{1}} \mathbf{H} \mathbf{~ N M R}$ (500 $\mathbf{~ M H z ;}$ $\left.\mathrm{CDCl}_{3}\right): \delta 7.26-7.10(\mathrm{~m}, 5 \mathrm{H}), 6.90(\mathrm{~s}, 1 \mathrm{H}) 6.81(\mathrm{~s}, 1 \mathrm{H}), 4.38(\mathrm{bs}, 1 \mathrm{H}), 4.24(\mathrm{q}, J=7.2 \mathrm{~Hz}, 1 \mathrm{H}), 2.24(\mathrm{~s}, 3 \mathrm{H})$, $2.12(\mathrm{~s}, 3 \mathrm{H}), 1.59(\mathrm{~d},, J=7.2 \mathrm{~Hz}, 3 \mathrm{H})$; LRMS (ESI): $\mathrm{m} / \mathrm{z}$ calcd for $\mathrm{C}_{16} \mathrm{H}_{18} \mathrm{O}[\mathrm{M}]^{+}:$:226.1; found: 226.1

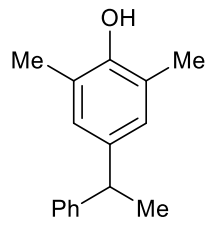

2,6-Dimethyl-4-(1-phenylethyl)phenol (6): Following the general procedures using 2,6-dimethylphenol (107 $\mathrm{mg}, 0.876 \mathrm{mmol}$ ) and purified by column chromatography to afford $168 \mathrm{mg}$ ( $85 \%$ yield) of a pale yellow oil. The analytical data for this compound matched with the previously reported compound ${ }^{4}$ : ${ }^{\mathbf{H}} \mathbf{H} \mathbf{~ N M R}(\mathbf{5 0 0} \mathbf{~ M H z}$; $\left.\mathrm{CDCl}_{3}\right): \delta 7.31-7.10(\mathrm{~m}, 5 \mathrm{H}), 6.82(\mathrm{~s}, 2 \mathrm{H}), 4.44(\mathrm{bs}, 1 \mathrm{H}), 4.02(\mathrm{q}, J=7.2 \mathrm{~Hz}, 1 \mathrm{H}), 2.20(\mathrm{~s}, 6 \mathrm{H}), 1.59(\mathrm{~d}, J=7.2$ $\mathrm{Hz}, 3 \mathrm{H}$ ); LRMS (ESI): m/z calcd for $\mathrm{C}_{16} \mathrm{H}_{18} \mathrm{O}[\mathrm{M}]^{+}:$: 226.1; found: 226.1<smiles>[M]C([O-])(c1ccccc1)c1cccc(C(C)C)c1O</smiles>

and<smiles>CC(C)c1cc(C(C)c2ccccc2)ccc1O</smiles> 
2-(1-Phenethyl)-6-(propan-2-yl)phenol (7a) and 4-(1-phenethyl)-2-(propan-2-yl)phenol (7b): (mixture of approximately $2: 1$ ortho and para isomers): Following the general procedures using 2-isopropylphenol (119 $\mathrm{mg}, 0.874 \mathrm{mmol}$ ) and purified by column chromatography to afford $158 \mathrm{mg}$ (73\% combined yield) of a colorless oil. Analytical data: ${ }^{1} \mathbf{H}$ NMR (500 $\mathbf{~ M H z} \mathbf{C D C l}_{3}$ ): $\delta$ (major isomer, 7a) 7.30 - 7.11 (m, $7 \mathrm{H}$ ), 6.97 - 6.94 $(\mathrm{d}, J=8 \mathrm{~Hz}, 1 \mathrm{H}$ ), 4.60 (bs, $1 \mathrm{H}), 4.28(\mathrm{q}, J=7.2 \mathrm{~Hz}, 1 \mathrm{H}), 3.14$ (sept, $J=8 \mathrm{~Hz}, 1 \mathrm{H}), 1.63(\mathrm{~d}, J=7.1 \mathrm{~Hz}, 3 \mathrm{H}), 1.21$ (dd, $J=7.1 \mathrm{~Hz}, 6 \mathrm{H}$ ); (minor isomer, 7b) $7.33-7.11(\mathrm{~m}, 7 \mathrm{H}), 6.65(\mathrm{~d}, J=7.5 \mathrm{~Hz}, 1 \mathrm{H}), 4.50(\mathrm{bs}, 1 \mathrm{H}), 4.08(\mathrm{q}, J=$ $7.2 \mathrm{~Hz}, 1 \mathrm{H}$ ), 3.14 (sept, $J=7.1 \mathrm{~Hz}, 1 \mathrm{H}), 1.60(\mathrm{~d}, J=7.2 \mathrm{~Hz}, 3 \mathrm{H}), 1.24(\mathrm{~d}, J=7.1 \mathrm{~Hz}, 6 \mathrm{H}) ;{ }^{13} \mathrm{C}$ NMR (75 MHz, $\mathrm{CDCl}_{3}$ ): $\delta$ major isomer (7a): 150.8, 145.2, 135.0, 131.3, 129.0, 127.6, 126.8, 125.2, 124.4, 120.6, 39.7, 27.1, 23.1, 22.8, 21.7; minor isomer (7b): 152.7, 149.0, 134.2, 128.3, 127.5, 126.7, 126.5, 125.9, 121.1, 115.3, 44.8, 27.4, 22.6, 22.6, 21.6; IR (thin film neat, $\mathrm{cm}^{-1}$ ): 3537, 2964, 2876, 1502, 1450, 1253, 821, 750, 700; HRMS (ESI): $\mathrm{m} / \mathrm{z}$ calcd for $\mathrm{C}_{17} \mathrm{H}_{21} \mathrm{O}[\mathrm{M}+\mathrm{H}]^{+}: 241.1593$; found 241.1630<smiles>CC(C)c1ccc(C(C)C)c(O)c1</smiles>

5-(1-Methylethyl)-2-(1-phenylethyl)phenol (8): Following the general procedures using 3-isopropylphenol (119 $\mathrm{mg}, 0.874 \mathrm{mmol}$ ) and purified by column chromatography to afford $147 \mathrm{mg}$ (70\% yield) of a colorless oil. Analytical data: ${ }^{1} \mathbf{H}$ NMR (500 $\left.\mathbf{~ M H z ; ~} \mathbf{C D C l}_{3}\right): \delta 7.31-7.28(\mathrm{~m}, 3 \mathrm{H}), 7.26-7.25(\mathrm{~m}, 1 \mathrm{H}), 7.21-7.20(\mathrm{~m}, 1 \mathrm{H})$, $7.16(\mathrm{~d}, J=7.8 \mathrm{~Hz}, 1 \mathrm{H}), 6.81(\mathrm{~d}, J=7.8 \mathrm{~Hz}, 1 \mathrm{H}), 6.63(\mathrm{~s}, 1 \mathrm{H}), 4.51$ (bs, $1 \mathrm{H}), 4.31$ (q, J=7.3 Hz, $1 \mathrm{H}$ ), 2.83 (sept, $J=6.8 \mathrm{~Hz}, 1 \mathrm{H}), 1.62(\mathrm{~d}, J=7.3 \mathrm{~Hz}, 3 \mathrm{H}), 1.22(\mathrm{~d}, J=6.8 \mathrm{~Hz}, 6 \mathrm{H}) ;{ }^{13} \mathrm{C}$ NMR $\left(75 \mathbf{~ M H z} \mathrm{CDCl}_{3}\right): \delta$ 153.2, 148.7, 145.6, 129.1, 128.7, 127.7, 127.6, 126.4, 119.0, 114.2, 38.8, 33.8, 24.1, 21.3; IR (thin film neat, $\mathrm{cm}^{-1}$ ): 3455, 2924, 2852, 1575, 1446, 1292, 1251, 958, 84, 752, 709; HRMS (ESI): m/z calcd for $\mathrm{C}_{17} \mathrm{H}_{21} \mathrm{O}[\mathrm{M}+\mathrm{H}]^{+}: 241.1593$; found 241.1620 .<smiles>COc1ccc(C(C)c2ccccc2)c(OC)c1OC</smiles>

1,2,3-Trimethoxy-4-(1-phenylethyl)-benzene (9): Following the general procedures using 1,2,3trimethoxybenzene $(147 \mathrm{mg}, 0.874 \mathrm{mmol}$ ) and purified by column chromatography to afford $176 \mathrm{mg}$ (74 \% yield) of a colorless oil. The analytical data for this compound matched with the previously reported compound ${ }^{5}$ : ${ }^{1} \mathbf{H}$ NMR (500 MHz; $\left.\mathbf{C D C l}_{3}\right): \delta 7.29-7.15(\mathrm{~m}, 5 \mathrm{H}), 6.89(\mathrm{~d}, J=8.7 \mathrm{~Hz}, 1 \mathrm{H}), 6.64(\mathrm{~d}, \mathrm{~J}=8.7 \mathrm{~Hz}, 1$ $\mathrm{H}), 4.45(\mathrm{q}, J=7.2 \mathrm{~Hz}, 1 \mathrm{H}), 3.81-3.85(\mathrm{~m}, 9 \mathrm{H}), 1.56(\mathrm{~d}, J=7.2 \mathrm{~Hz}, 1 \mathrm{H}) ;$ LRMS (ESI): $\mathrm{m} / \mathrm{z}$ calcd for $\mathrm{C}_{17} \mathrm{H}_{20} \mathrm{O}_{3}$ $[\mathrm{M}]^{+}: 272.1$; found: 272.1

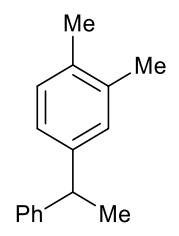

1,2-Dimethyl-4-(1-phenylethyl)-benzene (10): Following the general procedures using o-xylene $(93 \mathrm{mg}$, $0.876 \mathrm{mmol}$ ) and purified by column chromatography to afford $101 \mathrm{mg}$ (55\% yield) of a colorless oil. The analytical data for this compound matched with the previously reported compound ${ }^{1}$ : ${ }^{\mathbf{1}} \mathbf{H} \mathbf{~ N M R}(\mathbf{5 0 0} \mathbf{~ M H z}$; 
$\mathrm{CDCl}_{3}$ ): $\delta 7.27-6.90(\mathrm{~m}, 8 \mathrm{H}), 4.30(\mathrm{q}, J=7.2 \mathrm{~Hz}, 1 \mathrm{H}), 2.37-2.20(\mathrm{~m}, 6 \mathrm{H}), 1.60(\mathrm{~d}, J=7.2 \mathrm{~Hz}, 3 \mathrm{H})$; LRMS (ESI): $\mathrm{m} / \mathrm{z}$ calcd for $\mathrm{C}_{16} \mathrm{H}_{18}[\mathrm{M}]^{+}:$: 210.1; found: 210.1

\section{Procedure for the Hydroarylation of Styrene with 1-Methylindole}

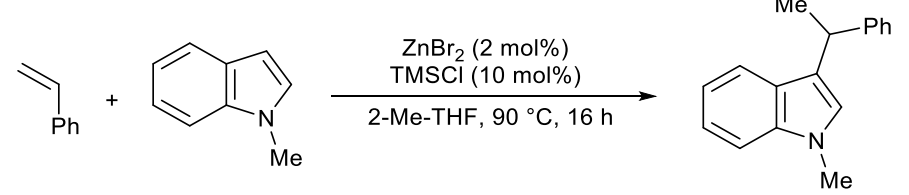

1-Methyl-3-(1-phenylethyl)-1H-Indole (11): To a $4 \mathrm{~mL}$ screw-capped vial with $\mathrm{ZnBr}_{2}$ (4 mg, $0.018 \mathrm{mmol}$ ) open to air, 2-methyltetrahydrofuran $(800 \mu \mathrm{L})$ and chlorotrimethylsilane $(12 \mu \mathrm{L}, 0.095 \mathrm{mmol})$ were added. 1Methylindole $(109 \mu \mathrm{L}, 0.873 \mathrm{mmol})$ was then added followed by styrene $(100 \mu \mathrm{L}, 0.872 \mathrm{mmol})$. The reaction mixture was stirred at $90{ }^{\circ} \mathrm{C}$ for 16 hours. A $10 \mu \mathrm{L}$ aliquot of this solution was removed for GC-MS analysis. The rest of the solution was filtered through a pad of celite $(1 \mathrm{~cm}, \mathrm{DCM}$ wash) and the solvent was evaporated to dryness. The crude reaction mixture was purified by column chromatography (hexane:EtOAc = 9:1) to obtain the $72 \mathrm{mg}$ ( $35 \%$ yield) of a pale yellow oil. The analytical data for this compound matched with the previously reported compound ${ }^{6}{ }^{1} \mathbf{H}$ NMR $\left(500 \mathbf{~ M H z} \mathbf{C D C l}_{3}\right): \delta 7.38(\mathrm{~d}, J=8.2 \mathrm{~Hz}, 1 \mathrm{H}), 7.31-7.19(\mathrm{~m}$, 7H), 7.09 (t, $J=7.0 \mathrm{~Hz}, 1 \mathrm{H}), 6.85(\mathrm{~s}, 1 \mathrm{H}), 4.38(\mathrm{q}, J=7.2 \mathrm{~Hz}, 1 \mathrm{H}), 3.77(\mathrm{~s}, 3 \mathrm{H}), 1.71(\mathrm{~d}, J=7.2 \mathrm{~Hz}, 3 \mathrm{H})$; LRMS (ESI): $\mathrm{m} / \mathrm{z}$ calcd for $\mathrm{C}_{17} \mathrm{H}_{17} \mathrm{~N}[\mathrm{M}]^{+}: 235.1$; found: 235.1<smiles>COc1ccc(C(C)c2ccc(C)cc2)cc1</smiles>

1-Methoxy-4-[1-(4-methylphenyl)ethyl]benzene (12): Following the general procedure of styrene with anisole using 4-methylstyrene $(95 \mu \mathrm{L}, 0.896 \mathrm{mmol})$ and purified by column chromatography to afford $193 \mathrm{mg}$ (95\% combined yield) of a colorless oil. The analytical data for this compound matched with the previously reported compound ${ }^{1}:{ }^{1} \mathbf{H}$ NMR $\left(500 ~ \mathbf{M H z}_{\mathbf{C}} \mathbf{C D C l}_{3}\right): \delta 7.26-7.11(\mathrm{~m}, 6 \mathrm{H}), 6.84(\mathrm{~d}, J=2.1 \mathrm{~Hz}, 2 \mathrm{H}), 4.11(\mathrm{q}, J=$ $6.3 \mathrm{~Hz}, 1 \mathrm{H}$ ), $3.79(\mathrm{~s}, 3 \mathrm{H}), 2.33(\mathrm{~s}, 3 \mathrm{H}), 1.58(\mathrm{~d}, J=6.3 \mathrm{~Hz}, 3 \mathrm{H})$; LRMS (ESI): m/z calcd for $\mathrm{C}_{16} \mathrm{H}_{18} \mathrm{O}$ [M] ${ }^{+}: 226.1$; found: 226.1<smiles>COc1ccc(C(C)c2ccc(F)cc2)cc1</smiles>

and

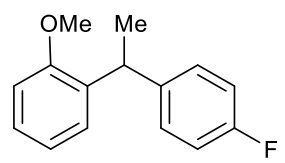

1-Methoxy-4-[1-(4-fluorophenyl)ethyl]benzene (13a) and 1-methoxy-2-[1-(4-fluorophenyl)ethyl]benzene (13b) (mixture of approximately $8: 1$ para and ortho isomers): Following the general procedure of styrene with anisole using 4-fluorostyrene $(107 \mu \mathrm{L}, 0.898 \mathrm{mmol})$ and purified by column chromatography to afford $172 \mathrm{mg}(83 \%$ combined yield) of a colorless oil. The analytical data for this compound matched with the 
previously reported compound ${ }^{1}$ : ${ }^{1} \mathbf{H}$ NMR (500 $\left.\mathbf{~ M H z ; ~} \mathbf{C D C l}_{3}\right): \delta$ (major isomer, 13a) $7.22-7.09(\mathrm{~m}, 4 \mathrm{H}), 6.97$ $-6.94(\mathrm{~m}, 2 \mathrm{H}), 6.82(\mathrm{~d}, J=6.5 \mathrm{~Hz}, 2 \mathrm{H}), 4.11(\mathrm{q}, J=5.4 \mathrm{~Hz}, 1 \mathrm{H}), 3.80(\mathrm{~s}, 3 \mathrm{H}), 1.61(\mathrm{~d}, J=5.4 \mathrm{~Hz}, 3 \mathrm{H})$; (minor isomer, 13b) $7.22-7.09(\mathrm{~m}, 4 \mathrm{H}), 6.95-6.92(\mathrm{~m}, 2 \mathrm{H}), 6.80(\mathrm{~d}, J=6.5 \mathrm{~Hz}, 2 \mathrm{H}), 4.56(\mathrm{q}, J=5.4 \mathrm{~Hz}, 1 \mathrm{H}), 3.79(\mathrm{~s}$, $3 \mathrm{H}), 1.58\left(\mathrm{~d}, J=5.4 \mathrm{~Hz}, 3 \mathrm{H}\right.$ ); LRMS (ESI): $\mathrm{m} / \mathrm{z}$ calcd for both isomers $\mathrm{C}_{15} \mathrm{H}_{15} \mathrm{FO}[\mathrm{M}]^{+}$: 230.1 ; found: 230.1<smiles>CCC(c1ccccc1)c1ccc(OC)cc1</smiles>

1-Methoxy-4-(1-phenylpropyl)benzene (14a) and 1-methoxy-2-(1-phenylpropyl)benzene (14b) (mixture of approximately 10:1 para and ortho isomers: Following the general procedure of styrene with anisole using $\beta$ methylstyrene $(97 \mu \mathrm{L}, 0.901 \mathrm{mmol}$ ) and purified by column chromatography to afford $104 \mathrm{mg}$ ( $51 \%$ yield) of a colorless oil. The analytical data for this compound matched with the previously reported compound ${ }^{1}:{ }^{\mathbf{1}} \mathbf{H}$ NMR (500 MHz; $\left.\mathbf{C D C l}_{3}\right): \delta$ (major isomer, 14a) $7.26-7.12(\mathrm{~m}, 7 \mathrm{H}), 6.83(\mathrm{~d}, J=6.4 \mathrm{~Hz}, 2 \mathrm{H}), 3.72-3.74(\mathrm{~m}, 4$ $\mathrm{H}), 2.04(\mathrm{~m}, 2 \mathrm{H}), 0.88(\mathrm{~m}, 3 \mathrm{H})$; (minor isomer, 14b) $7.26-7.12(\mathrm{~m}, 7 \mathrm{H}), 6.80(\mathrm{~d}, J=6.2 \mathrm{~Hz}, 2 \mathrm{H}), 4.28(\mathrm{t}, J=$ $7.2 \mathrm{~Hz}, 1 \mathrm{H}), 3.72-3.70(\mathrm{~m}, 3 \mathrm{H}), 2.00(\mathrm{~m} 2 \mathrm{H}), 0.87(3 \mathrm{H})$;LRMS (ESI): $\mathrm{m} / \mathrm{z}$ calcd for both isomers $\mathrm{C}_{16} \mathrm{H}_{18} \mathrm{O}[\mathrm{M}]^{+}$: 226.1; found: 226.1

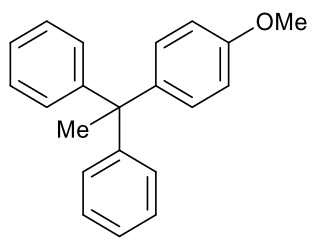

1-(1,1-Diphenylethyl)-4-methoxybenzene (15): Following the general procedure of styrene with anisole using diphenylethylene $(166 \mu \mathrm{L}, 0.902 \mathrm{mmol})$ and purified by column chromatography to afford $246 \mathrm{mg}$ ( $95 \%$ yield) of a colorless oil which crystallized upon standing. The analytical data for this compound matched with the previously reported compound ${ }^{1}$ : ${ }^{1} \mathbf{H}$ NMR (500 MHz; $\left.\mathbf{C D C l}_{3}\right): \delta 7.36\left(\mathrm{td}, J_{1}=5.5 \mathrm{~Hz}, J_{2}=1.5 \mathrm{~Hz}, 4 \mathrm{H}\right), 7.34$ $\left(\mathrm{dd}, J_{1}=5.5 \mathrm{~Hz}, J_{2}=1.5 \mathrm{~Hz}, 2 \mathrm{H}\right), 7.20\left(\mathrm{dd}, J_{1}=5.5 \mathrm{~Hz}, J=1.5 \mathrm{~Hz}, 4 \mathrm{H}\right), 7.10(\mathrm{dd}, J=5.5 \mathrm{~Hz}, J=1.5 \mathrm{~Hz}, 2 \mathrm{H}), 6.88$ (dd, $J=5.5 \mathrm{~Hz}, J=1.5 \mathrm{~Hz}, 2 \mathrm{H}$ ), $3.86(\mathrm{~s}, 3 \mathrm{H}), 2.26(\mathrm{~s}, 3 \mathrm{H})$; LRMS (ESI): m/z calcd for $\mathrm{C}_{21} \mathrm{H}_{20} \mathrm{O}[\mathrm{M}]^{+}: 288.1$; found: 288.1

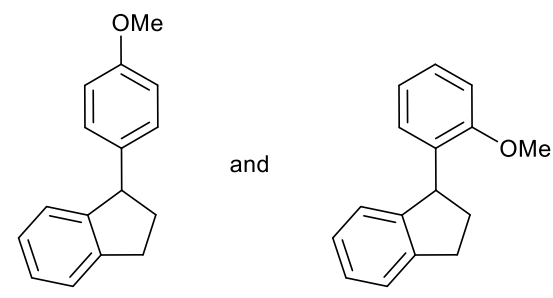

1-(4-Methoxyphenyl)-2,3-dihydro-1H-indene (16a) and 1-(2-methoxyphenyl)-2,3-dihydro-1H-indene (16b) (mixture of approximately 15:1 para and ortho isomers): Following the general procedure of styrene with anisole using indene $(104 \mu \mathrm{L}, 0.899 \mathrm{mmol})$ and purified by column chromatography to afford $127 \mathrm{mg}(63 \%$ combined yield) of a pale yellow oil. The analytical data for this compound matched with the previously reported compound ${ }^{1}$ : ${ }^{1} \mathbf{H}$ NMR (500 $\mathbf{M H z}^{\left.-\mathbf{C D C l}_{3}\right)}$ : $\delta$ (major isomer, 16a) $7.29(\mathrm{~d}, J=6.2 \mathrm{~Hz}, 1 \mathrm{H}), 7.20-7.11$ 
$(\mathrm{m}, 4 \mathrm{H}), 6.97(\mathrm{~d}, J=6.2 \mathrm{~Hz}, 1 \mathrm{H}), 6.87(\mathrm{~d}, J=6.2 \mathrm{~Hz}, 2 \mathrm{H}), 4.32(\mathrm{t}, J=6 \mathrm{~Hz}, 1 \mathrm{H}), 3.74(\mathrm{~s}, 3 \mathrm{H}), 3.02-2.89(\mathrm{~m}, 2$ $\mathrm{H}), 2.58-2.55(\mathrm{~m}, 1 \mathrm{H}), 2.05-2.00(\mathrm{~m}, 1 \mathrm{H})$; (minor isomer, 16b) $7.30(\mathrm{~d}, \mathrm{~d}, J=6.2 \mathrm{~Hz}, 1 \mathrm{H}) 7.20-7.11(\mathrm{~m}, 4 \mathrm{H})$, $6.96(\mathrm{~d}, J=6.2 \mathrm{~Hz}), 6.86(\mathrm{~d}, J=6.2 \mathrm{~Hz}, 2 \mathrm{H}), 4.70(\mathrm{t}, J=6 \mathrm{~Hz}, 1 \mathrm{H}), 3.02-2.89(\mathrm{~m}, 2 \mathrm{H}), 2.58-2.55(\mathrm{~m}, 1 \mathrm{H})$, $2.05-2.00(\mathrm{~m}, 1 \mathrm{H})$; LRMS (ESI): $\mathrm{m} / \mathrm{z}$ calcd for $\mathrm{C}_{16} \mathrm{H}_{16} \mathrm{O}[\mathrm{M}]^{+}:$: 224.1; found: 224.1

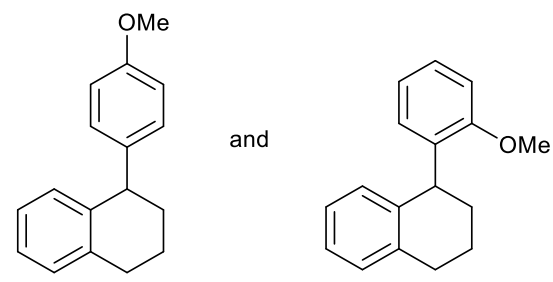

1-(4-Methoxyphenyl)-1,2,3,4-tetrahydronaphthalene (17a) and 1-(2-methoxyphenyl)-1,2,3,4tetrahydronaphthalene (17b) (mixture of approximately 15: 1 para and ortho isomers): Following the general procedure using 1,2-dihydronaphthalene (117 $\mu \mathrm{L}, 0.901 \mathrm{mmol})$ and purified by column chromatography to afford $126 \mathrm{mg}$ (59\% combined yield) of a pale yellow oil. The analytical data for this compound matched with the previously reported compound ${ }^{1}$ : $\left.{ }^{1} \mathbf{H} \mathbf{~ N M R ~ ( 5 0 0 ~} \mathbf{~ M H z} ; \mathbf{C D C l}_{3}\right): \delta$ (major isomer, 17a) $7.12-7.02(\mathrm{~m}, 6 \mathrm{H}), 6.86-6.83(\mathrm{~m}, 3 \mathrm{H}), 4.06(\mathrm{t}, J=6 \mathrm{~Hz}), 3.80(\mathrm{~s}, 3 \mathrm{H}), 2.91-2.79(\mathrm{~m}, 2 \mathrm{H}), 2.12-1.76$ $(\mathrm{m}, 4 \mathrm{H})$; (minor isomer, 17b) $7.12-7.02(\mathrm{~m}, 6 \mathrm{H}), 6.89-6.78(\mathrm{~m}, 2 \mathrm{H}), 4.61(\mathrm{t}, J=6 \mathrm{~Hz}), 2.91-2.79(\mathrm{~m}, 2 \mathrm{H})$, $2.12-1.76(\mathrm{~m}, 4 \mathrm{H})$; LRMS (ESI): $\mathrm{m} / \mathrm{z}$ calcd for $\mathrm{C}_{17} \mathrm{H}_{18} \mathrm{O}[\mathrm{M}]^{+}: 238.1$; found: 238.1

\section{General Procedure for the Hydroarylation of Alkyl Olefins with Anisole}

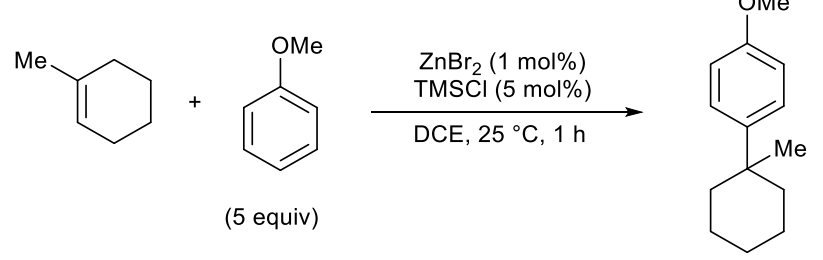

1-Methoxy-4-(1-methylcyclohexyl)-benzene (18): To a $4 \mathrm{~mL}$ screw-capped vial with $\mathrm{ZnBr}_{2}\left(2 \mathrm{mg}, 8.9 \times 10^{-3}\right.$ $\mathrm{mmol})$ open to air, 1,2-dichloroethane $(420 \mu \mathrm{L})$ and chlorotrimethylsilane $(6 \mu \mathrm{L}, 0.047 \mathrm{mmol})$ were added. Anisole $(475 \mu \mathrm{L}, 4.37 \mathrm{mmol})$ was then added followed by 1-methyl-1-cyclohexene (103 $\mu \mathrm{L}, 0.869 \mathrm{mmol})$. The reaction mixture was stirred at room temperature for 1 hour. A $10 \mu \mathrm{L}$ aliquot of this solution was removed for GC-MS analysis. The rest of the solution was filtered through a pad of celite (1 $\mathrm{cm}, \mathrm{DCM}$ wash) and the solvent was evaporated to dryness. The crude reaction mixture was purified by column chromatography (Hexane:EtOAc $=9: 1)$ to obtain the $125 \mathrm{mg}$ (70\% yield) of a pale yellow oil. The analytical data for this compound matched with the previously reported compound ${ }^{7}:{ }^{1} \mathbf{H} \mathbf{~ N M R}\left(500 \mathbf{~ M H z} ; \mathbf{C D C l}_{3}\right): \delta 7.29(\mathrm{~d}, J=8 \mathrm{~Hz}$, $2 \mathrm{H}), 6.87(\mathrm{~d}, J=8 \mathrm{~Hz}, 1 \mathrm{H}), 3.81(\mathrm{~s}, 3 \mathrm{H}), 1.99-1.94(\mathrm{~m}, 2 \mathrm{H}), 1.58-1.45(\mathrm{~m}, 8 \mathrm{H}), 1.17(\mathrm{~s}, 3 \mathrm{H})$; LRMS (ESI): $\mathrm{m} / \mathrm{z}$ calcd for $\mathrm{C}_{14} \mathrm{H}_{20} \mathrm{O}[\mathrm{M}]^{+}:$:204.1; found: 204.1 


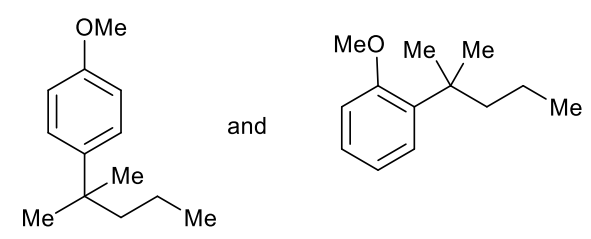

1-Methoxy-4-(2-methylpetan-2-yl)benzene (19a) and 1-methoxy-2-(2-methylpentan-2-yl)benzene (19b) (mixture of approximately 1.5:1 para and ortho isomers): Following the general procedure using 2-methyl-1pentene (108 $\mu \mathrm{L}, 0.875 \mathrm{mmol})$ and purified by column chromatography to afford $116 \mathrm{mg}$ (69\% yield) of a colorless oil. Analytical data: ${ }^{1} \mathbf{H}$ NMR $\left(500 \mathbf{~ M H z} \mathbf{C D C l}_{3}\right) \delta$ major isomer: $7.24(\mathrm{~d}, J=8.8 \mathrm{~Hz}, 2 \mathrm{H}), 6.84(\mathrm{~d}, J=8.8$ $\mathrm{Hz}, 2 \mathrm{H}), 3.79(\mathrm{~s}, 3 \mathrm{H}), 1.56-1.53(\mathrm{~m}, 2 \mathrm{H}), 1.27(\mathrm{~s}, 6 \mathrm{H}), 1.10-0.95(\mathrm{~m}, 2 \mathrm{H}), 0.81(\mathrm{t}, J=7.3 \mathrm{~Hz}, 3 \mathrm{H})$; minor isomer: $7.21-7.16(\mathrm{~m}, 2 \mathrm{H}), 6.90-6.86(\mathrm{~m}, 2 \mathrm{H}), 3.81(\mathrm{~s}, 3 \mathrm{H}), 1.79-1.75(\mathrm{~m}, 2 \mathrm{H}), 1.34(\mathrm{~s}, 6 \mathrm{H}), 1.10-0.95$ $(\mathrm{m}, 2 \mathrm{H}), 0.81(\mathrm{t}, J=7.3 \mathrm{~Hz}, 3 \mathrm{H}) .{ }^{13} \mathrm{C}$ NMR $\left(75 \mathrm{MHz}, \mathrm{CDCl}_{3}\right) \delta$ major isomer: 157.2, 142.0, 126.8, 113.4, 55.3, 47.4, 37.3, 29.3, 18.2, 15.0; minor isomer: 158.6, 137.0, 127.7, 127.0, 120.3, 111.6, 55.2, 43.7, 38.4, 28.6, 18.7, 15.1; IR (thin film neat, $\mathbf{c m}^{-1}$ ): 2957, 1612, 1514, 1249, 1238, 1037, 829, 748; HRMS (ESI): m/z calcd for $\mathrm{C}_{13} \mathrm{H}_{20} \mathrm{O}[\mathrm{M}]^{+}:$192.1513; found 192.1515 .

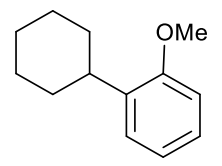

and<smiles>COc1ccc(C2CCCCC2)cc1</smiles>

1-Cyclohexyl-2-methoxybenzene (20a) and 1-cyclohexyl-4-methoxybenzene (20b) (mixture of approximately 1 : 1 para and ortho isomers): Following the general procedure using cyclohexene (88 $\mu \mathrm{L}$, $0.869 \mathrm{mmol}$ ) and purified by column chromatography to afford $18 \mathrm{mg}$ (11\% combined yield) of a colorless oil. The analytical data for this compound matched with the previously reported compound ${ }^{8}:{ }^{\mathbf{1}} \mathbf{H} \mathbf{~ N M R}(\mathbf{5 0 0}$ MHz; $\mathrm{CDCl}_{3}$ ): $\delta$ (major isomer, 20a) $7.12(\mathrm{~d}, J=8.0 \mathrm{~Hz}, 2 \mathrm{H}), 6.85(\mathrm{~d}, J=8 \mathrm{~Hz}, 2 \mathrm{H}), 3.80(\mathrm{~s}, 3 \mathrm{H}), 2.50-2.37(\mathrm{~m}$, $1 \mathrm{H}), 1.85-1.17(\mathrm{~m}, 10 \mathrm{H})$; (minor isomer, 20b) $7.12(\mathrm{~d}, J=8.0 \mathrm{~Hz}), 6.84(\mathrm{~d}, J=8.0 \mathrm{~Hz}), 3.83(\mathrm{~s}, 3 \mathrm{H}), 3.01-$ $2.90(\mathrm{~m}, 1 \mathrm{H}), 1.85-1.17(\mathrm{~m}, 10 \mathrm{H})$; LRMS (ESI): $\mathrm{m} / \mathrm{z}$ calcd for $\mathrm{C}_{13} \mathrm{H}_{18} \mathrm{O}[\mathrm{M}]^{+}:$190.1; found: 190.1

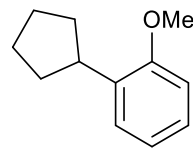

and

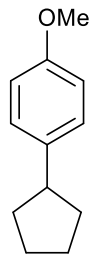

1-Cyclopentyl-2-methoxybenzene (21a) and 1-cyclopentyl-4-methoxybenzene (21b) (mixture of approximately 1 : 1 para and ortho isomers): Following the general procedures using cyclopentene $(80 \mu \mathrm{L}$, $0.874 \mathrm{mmol}$ ). Yield was determined by GC relative to dodecane as an internal standard. A crude ${ }^{1} \mathrm{H}$ NMR spectra was taken to verify the identity of the product. The analytical data for this compound matched with the previously reported compound ${ }^{9}:{ }^{1} \mathbf{H}$ NMR (500 $\left.\mathbf{~ M H z} ; \mathbf{C D C l}_{3}\right): \delta$ (major isomer) $7.16(\mathrm{~d}, J=8.0 \mathrm{~Hz}, 2 \mathrm{H})$, $6.83(\mathrm{~d}, J=8.0 \mathrm{~Hz}, 2 \mathrm{H}), 3.79(\mathrm{~s}, 3 \mathrm{H}), 2.97-2.92(\mathrm{~m}, 1 \mathrm{H}), 2.03-1.55(\mathrm{~m}, 8 \mathrm{H})$; (minor isomer) $7.25-7.16(\mathrm{~m}$, $2 \mathrm{H}), 6.95-6.94(\mathrm{~m}, 1 \mathrm{H}), 6.87(\mathrm{~d}, J=8.0 \mathrm{~Hz}), 3.83(\mathrm{~s}, 3 \mathrm{H}), 3.37-3.35(\mathrm{~m}, 1 \mathrm{H}), 2.03-1.55,8 \mathrm{H})$; LRMS (ESI): $\mathrm{m} / \mathrm{z}$ calcd for $\mathrm{C}_{12} \mathrm{H}_{16} \mathrm{O}[\mathrm{M}]^{+}: 176.1$; found: 176.1 


\section{References}

1 Chu, C.-M.; Huang, W.-J.; Liu, J.-T.; Yao, C.-F. Tetrahedron Lett. 2007, 48, 6881.

2 Kozlikovskii, Ya. B.; Chernyaev, B. V.; Litvin, A. L.; Kofanova, A. V. Zhurnal Org. Khimii 1986, 22, 1469.

3 Varghese, S.; Nagarajan, S.; Benzigar, M. R.; Mano, A.; ALOthman, Z. A.; Raj, G. A. G.; Vinu, A. Tetrahedron Lett. 2012, 53, 1485.

4 Hu, F.; Patel, M.; Luo, F.; Flach, C.; Mendelsohn, R.; Garfunkel, E.; He, H.; Szostak, M. J. Am. Chem. Soc. 2015, $137,14473$.

5 Sanz, R.; Alberto Martínez, Delia Miguel, Julia M. Álvarez-Gutiérrez, F. R. Adv. Synth. Catal. 2006, 348, 1841.

6 Zhao, X.; Yu, Z.; Xu, T.; Wu, P.; Yu, H. Org. Lett. 2007, 9, 5263.

7 Yamamoto, Y.; Itonaga, K. Chem. - A Eur. J. 2008, 14, 10705.

8 Nakamura, M.; Matsuo, K.; Ito, S.; Nakamura, E. J. Am. Chem. Soc. 2004, 126, 3686.

9 Duan, Z.; Li, W.; Lei, A. Org. Lett. 2016, 18, 4012. 


\section{NMR Spectra of New Compounds}

${ }^{1}$ H NMR for 2-(1-Methylethyl)-6-(1-phenylethyl)phenol and 2-(1-Methylethyl)-4-(1-phenylethyl)phenol (7a and 7b) PROTON

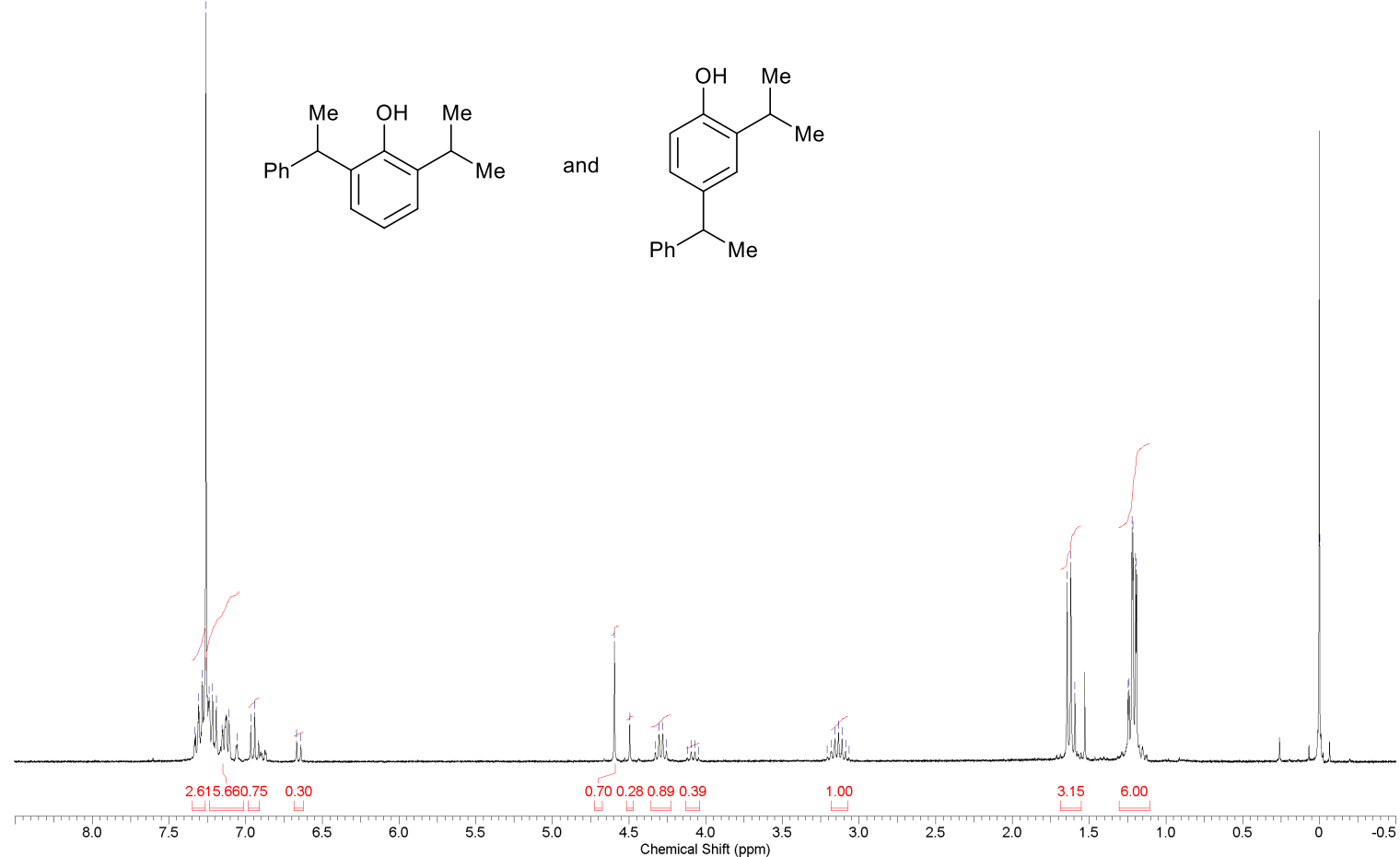

${ }^{13}$ C NMR for 2-(1-Methylethyl)-6-(1-phenylethyl)phenol and 2-(1-Methylethyl)-4-(1-phenylethyl)phenol (7a and 7b)<smiles>CC(C)c1cccc(C(C)(C)c2ccccc2)c1O</smiles>
and<smiles>CC(C)c1cc(C(C)c2ccccc2)ccc1O</smiles>
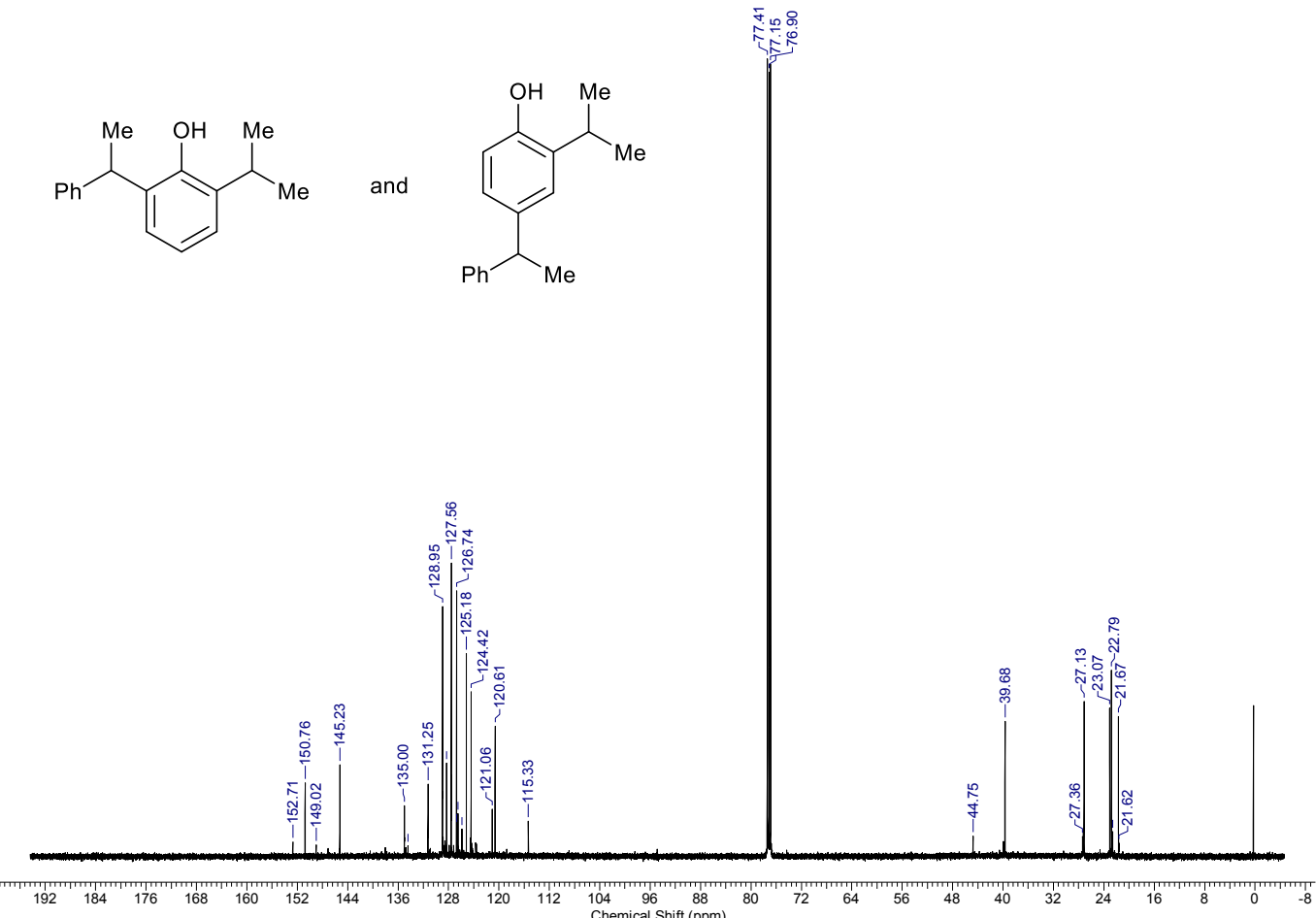
${ }^{1} \mathrm{H}$ NMR for 5-(1-Methylethyl)-2-(1-phenylethyl)phenol (8)

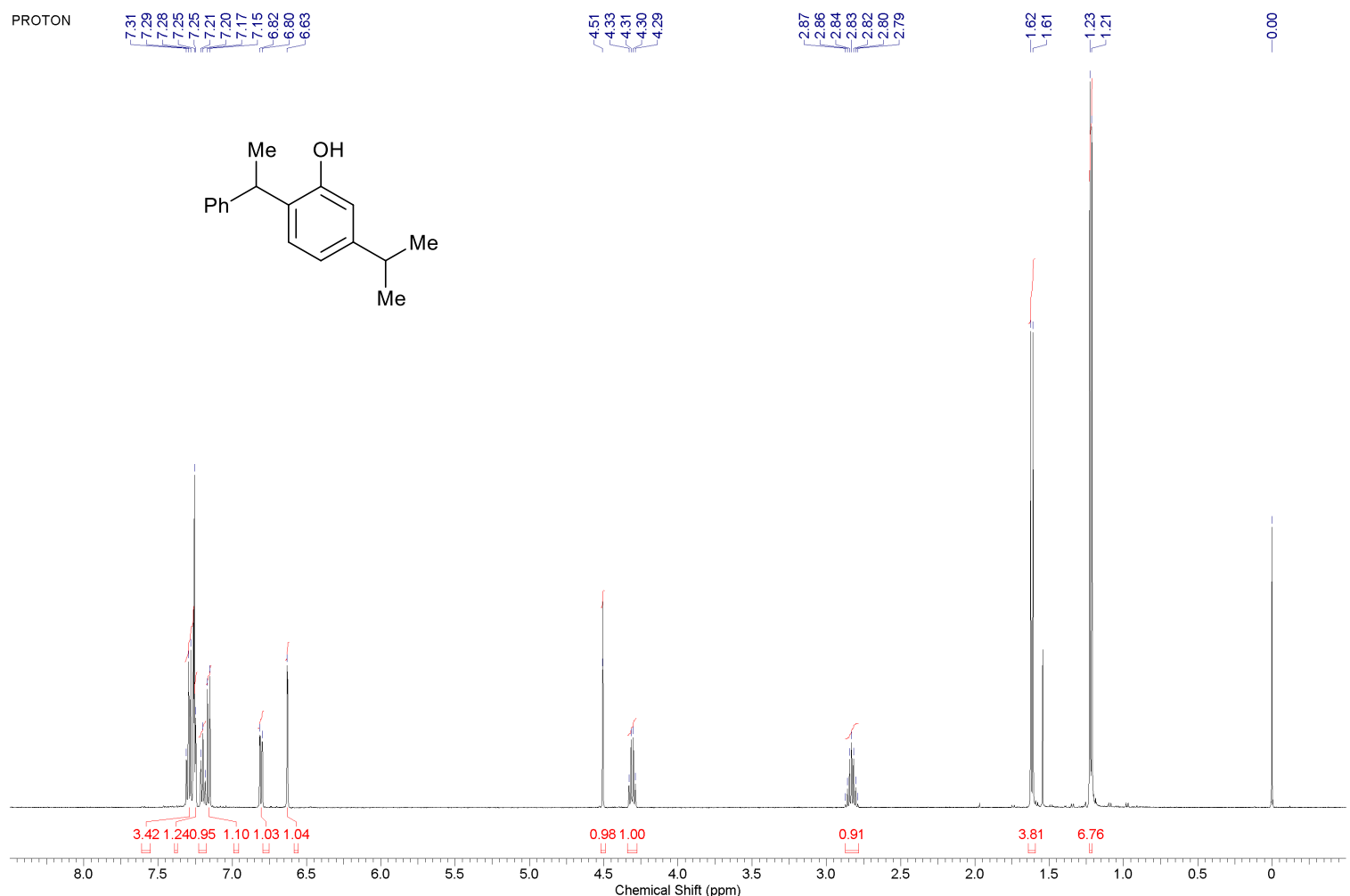

${ }^{13}$ C NMR for 5-(1-Methylethyl)-2-(1-phenylethyl)phenol (8)

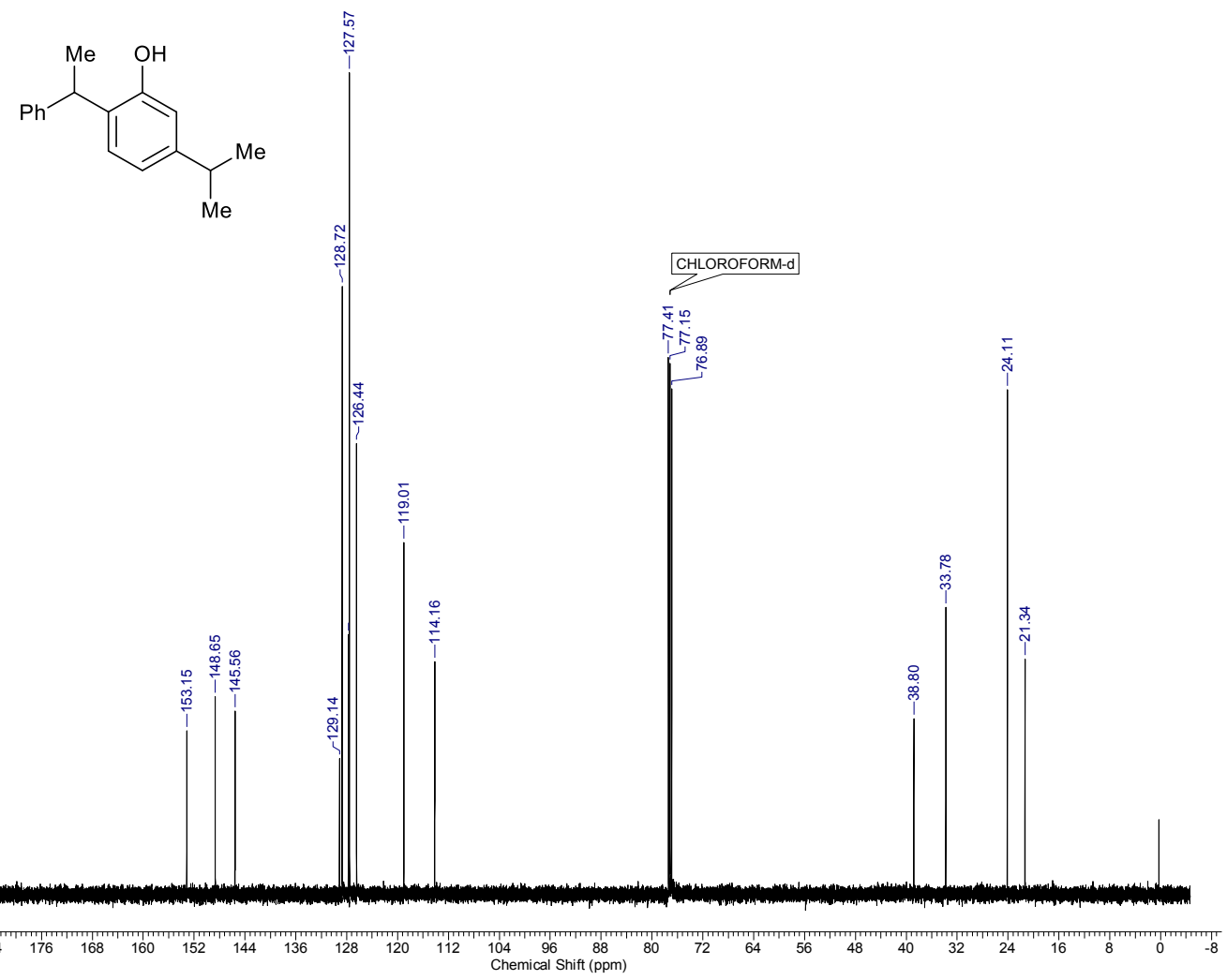


${ }^{1}$ H NMR for 1-(1,1-Dimethylbutyl)-4-methoxybenzene (19)

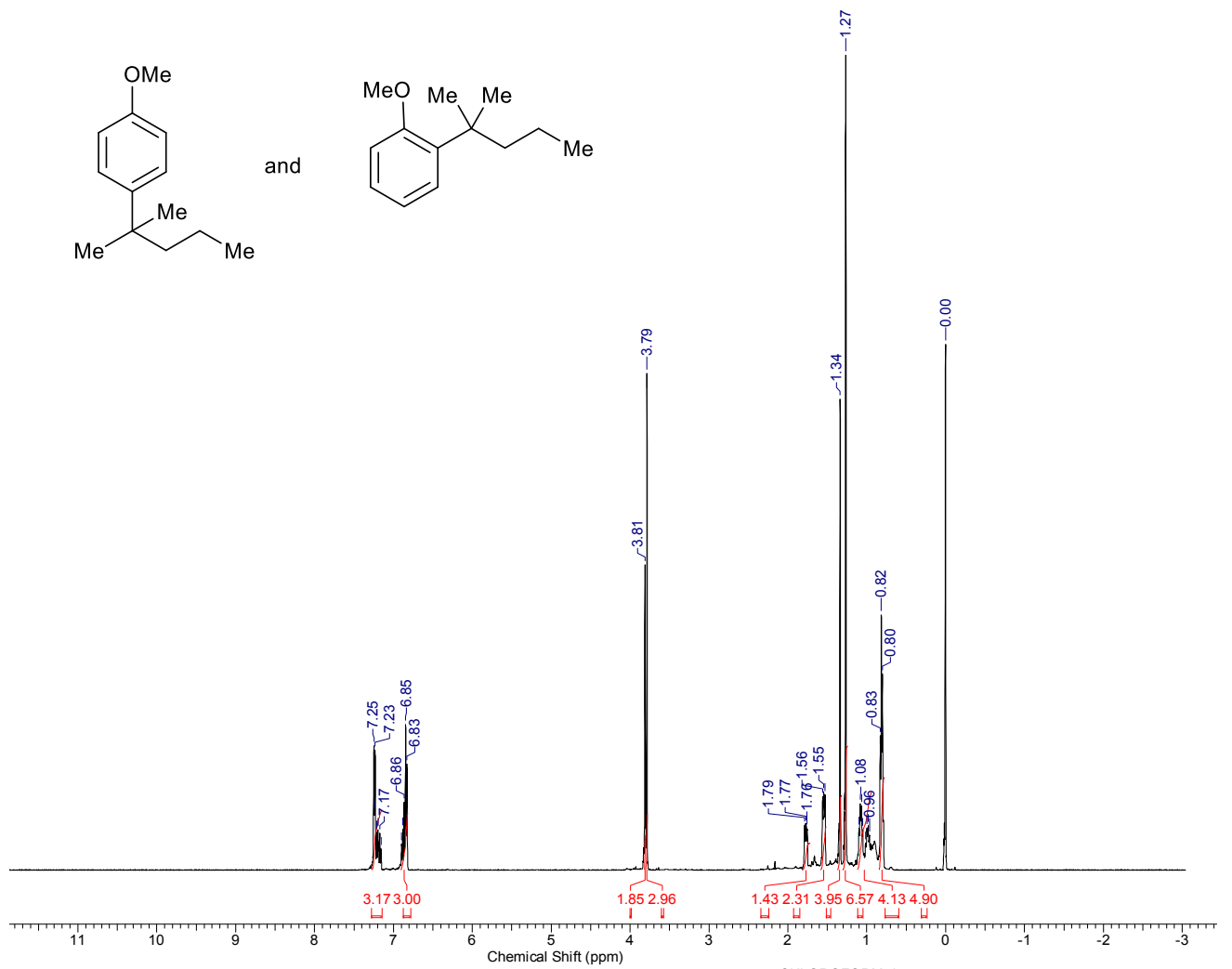

${ }^{13}$ C NMR for 1-(1,1-Dimethylbutyl)-4-methoxybenzene (19)

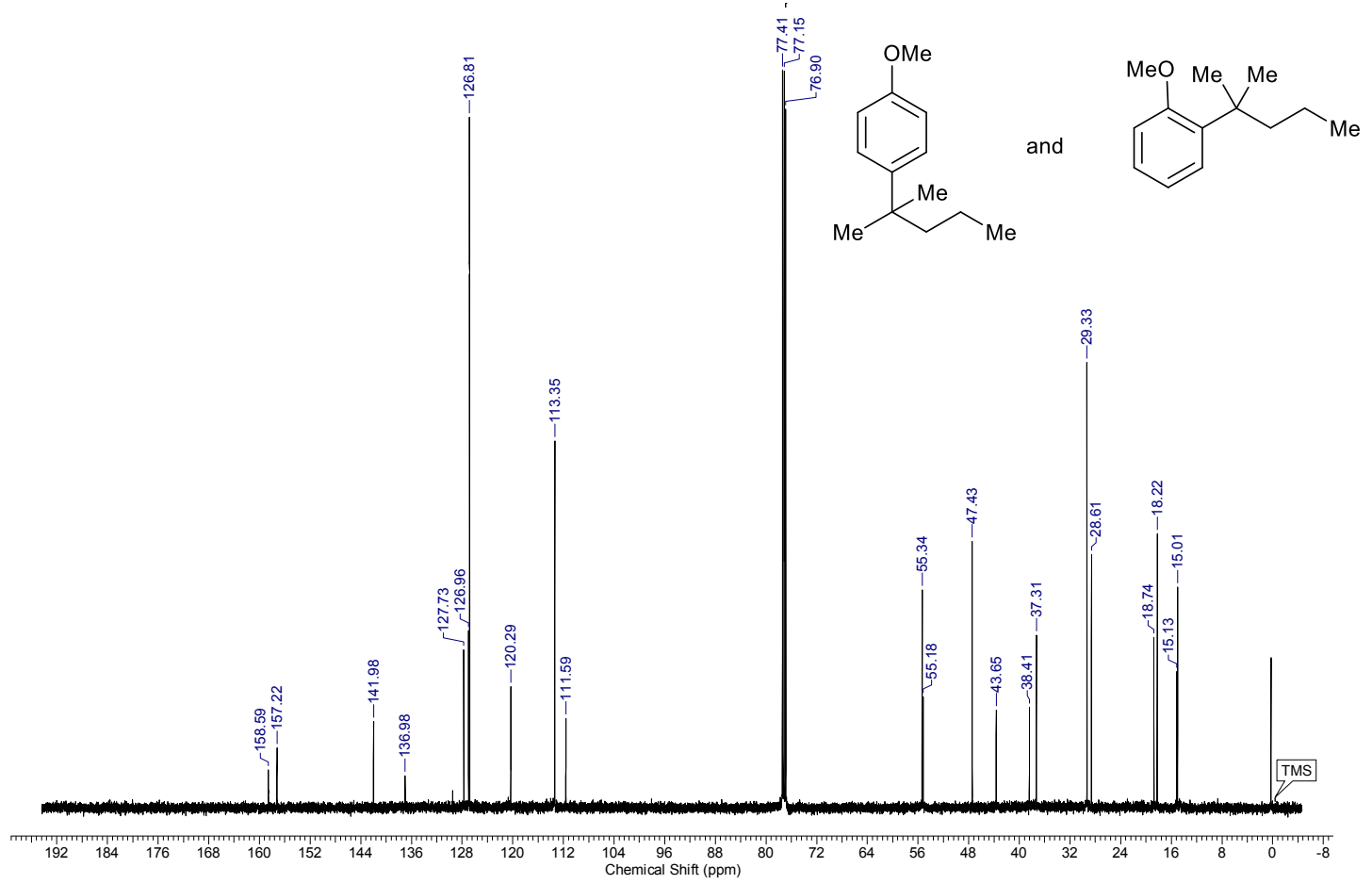




\section{Spectra of Previously Reported Compounds}

${ }^{1} \mathrm{H}$ NMR of 1-Methoxy-2-(1-phenylethyl)benzene (1a) and 1-methoxy-4-(1-phenylethyl)benzene (1b)

anisole.esp<smiles>COc1ccc(C(C)c2ccccc2)cc1</smiles>
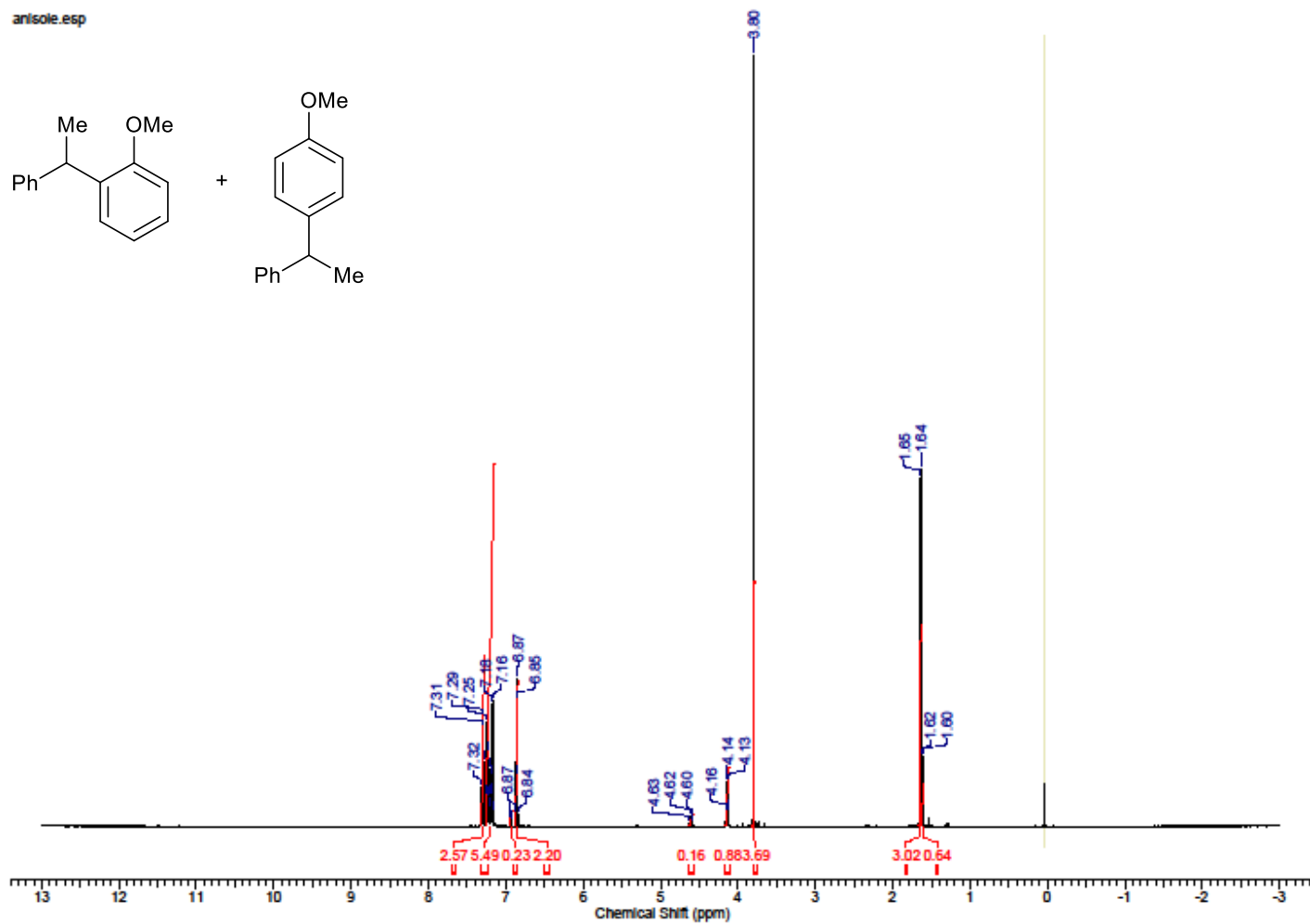

${ }^{1}$ H NMR of 2-Methyl-6-(1-phenylethyl)phenol (2a) and 2-methyl-4-(1-phenylethyl)phenol (2b) orthocresol.esp<smiles>Cc1cccc(C(C)c2ccccc2)c1O</smiles>

and<smiles>Cc1cc(C(C)c2ccccc2)ccc1O</smiles>
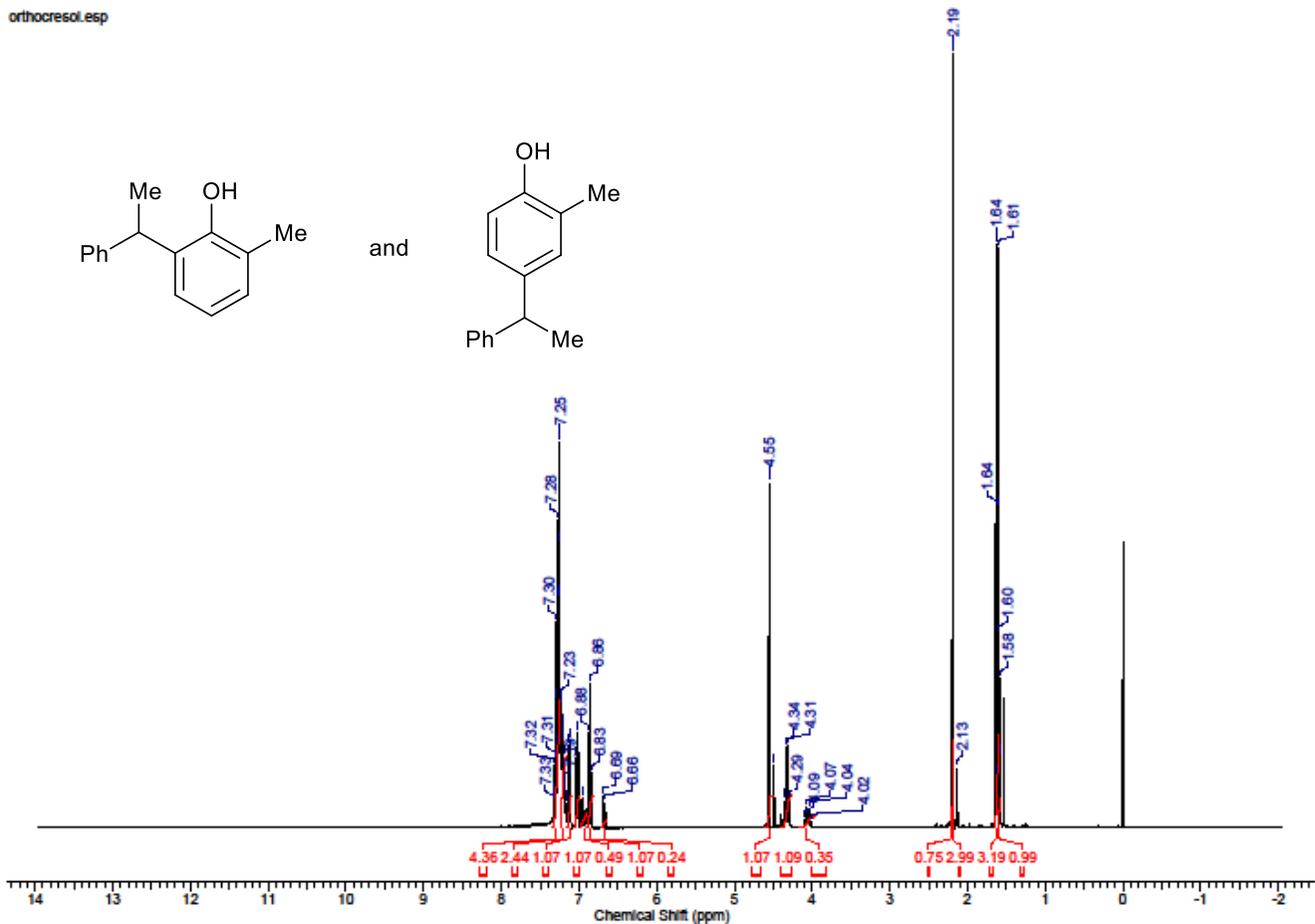
${ }^{1} \mathrm{H}$ NMR of 5-Methyl-2-(1-phenylethyl)phenol (3)

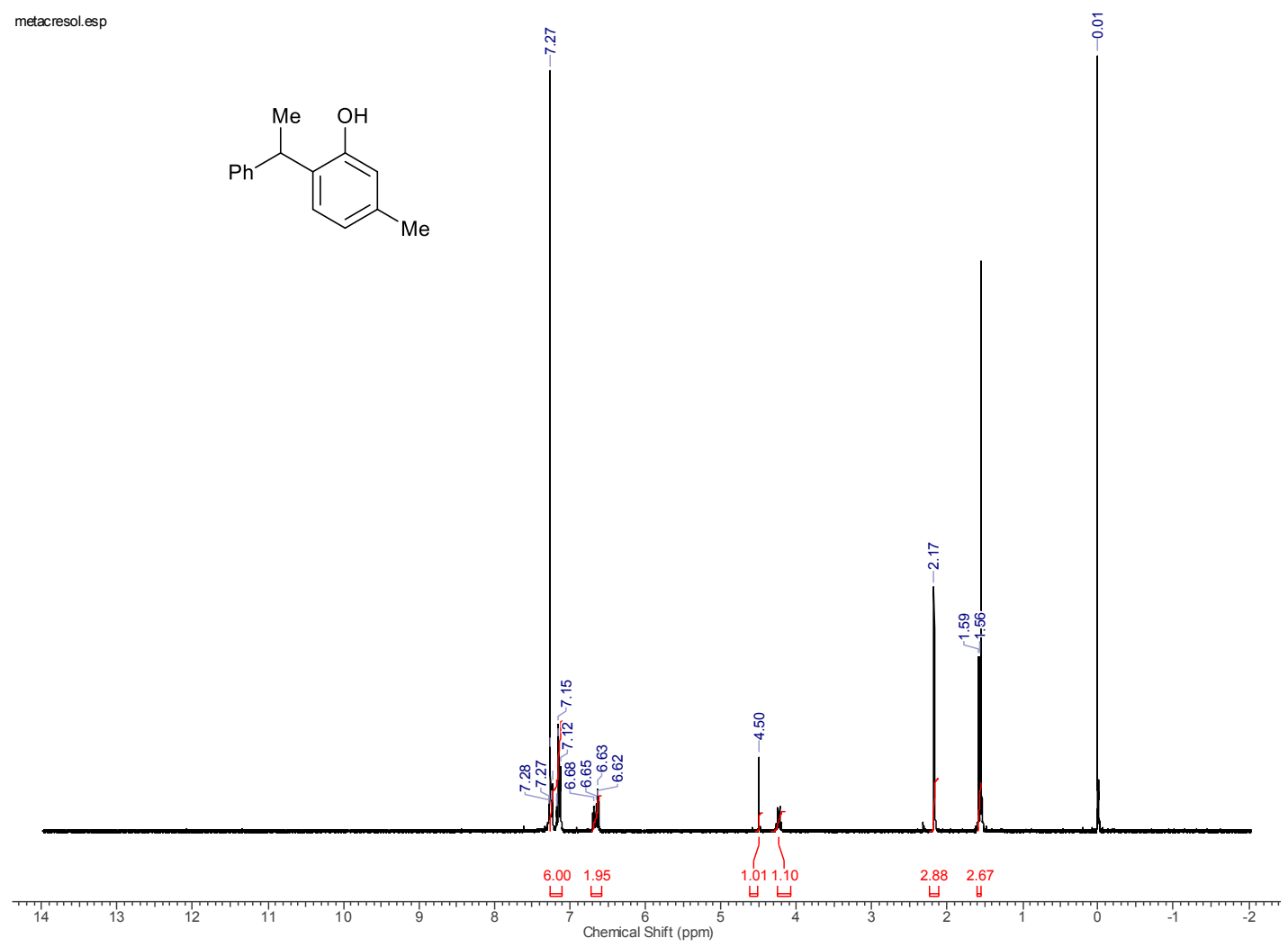

${ }^{1} \mathrm{H}$ NMR of 4-Methyl-2-(1-phenylethyl)phenol (4)

paracresol.esp

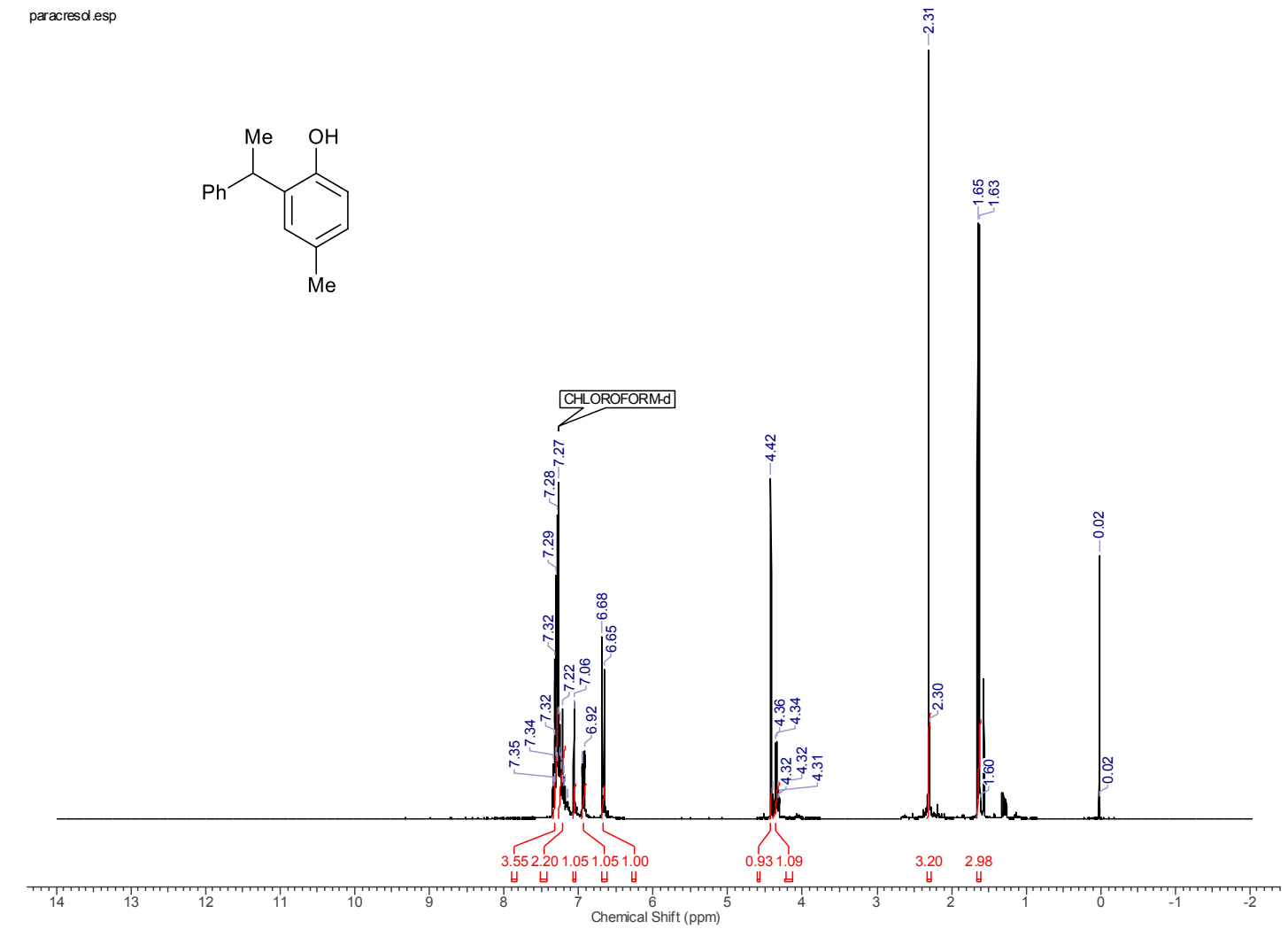


${ }^{1}$ H NMR of 2,4-Dimethyl-6-(1-phenylethyl)phenol (5)

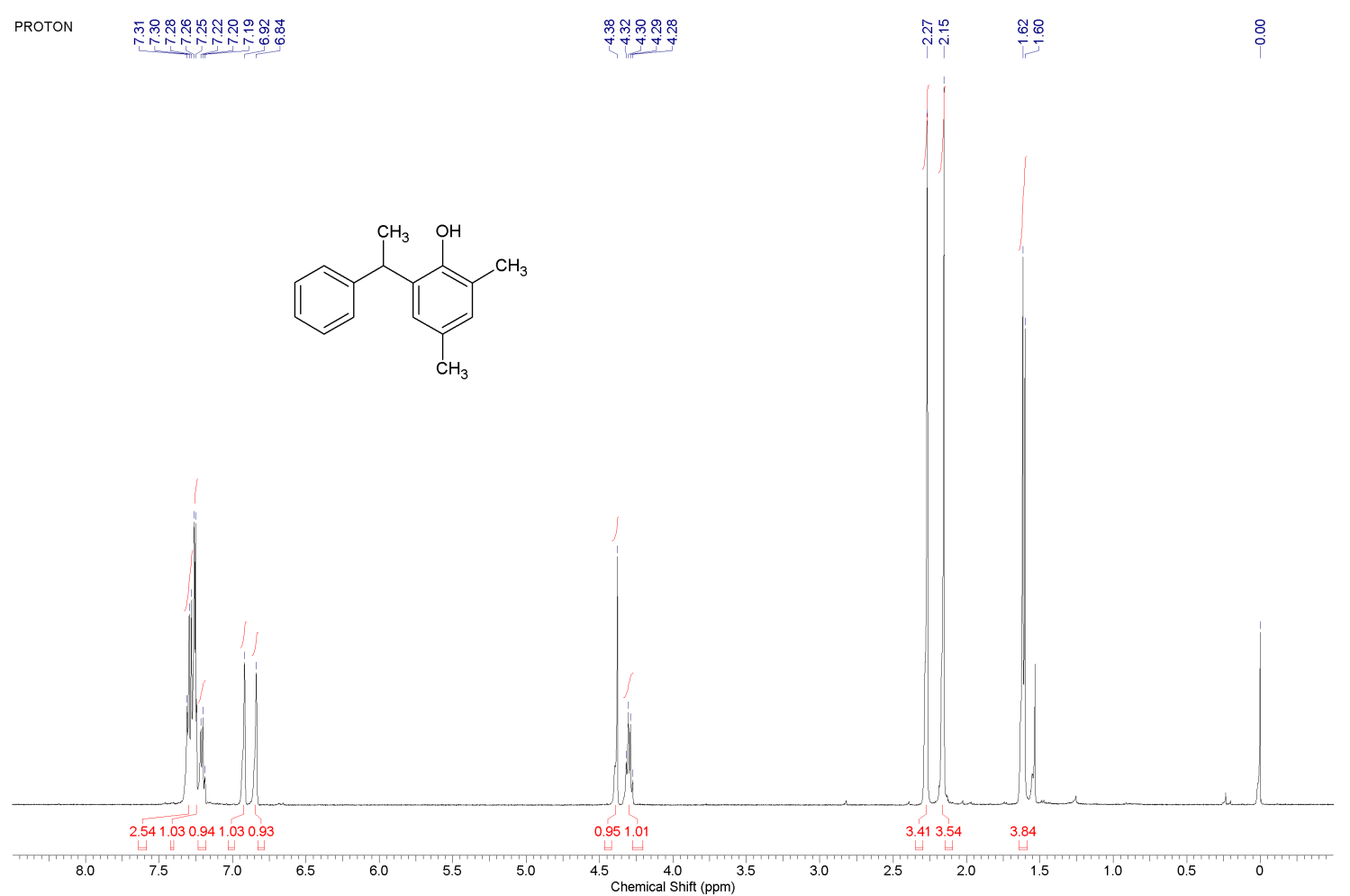

${ }^{1} \mathrm{H}$ NMR of 2,6-Dimethyl-4-(1-phenylethyl)phenol (6)

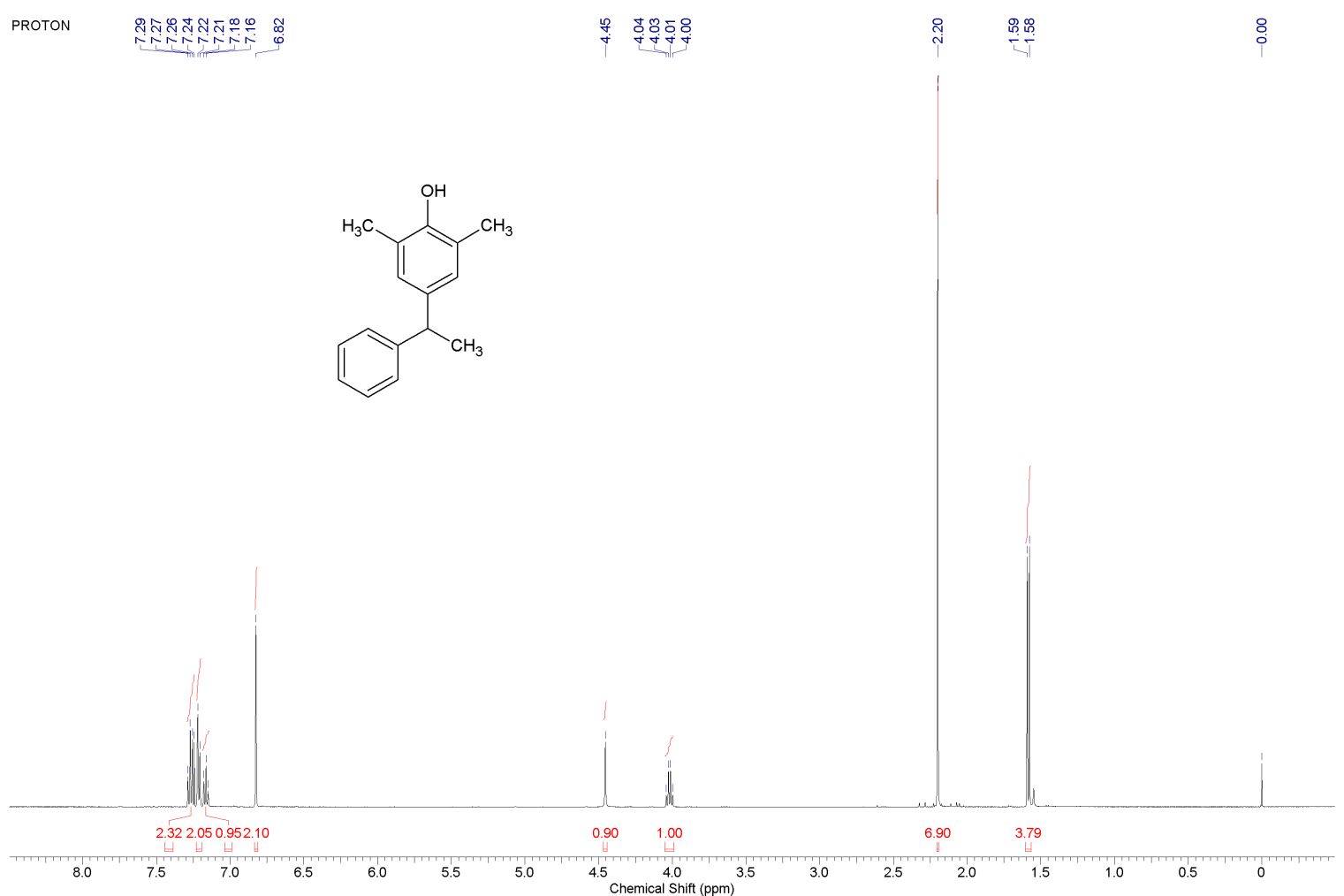


${ }^{1} \mathrm{H}$ NMR of 1,2,3-Trimethoxy-4-(1-phenylethyl)-benzene (9)

PROTON

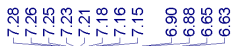

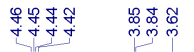

战号

:

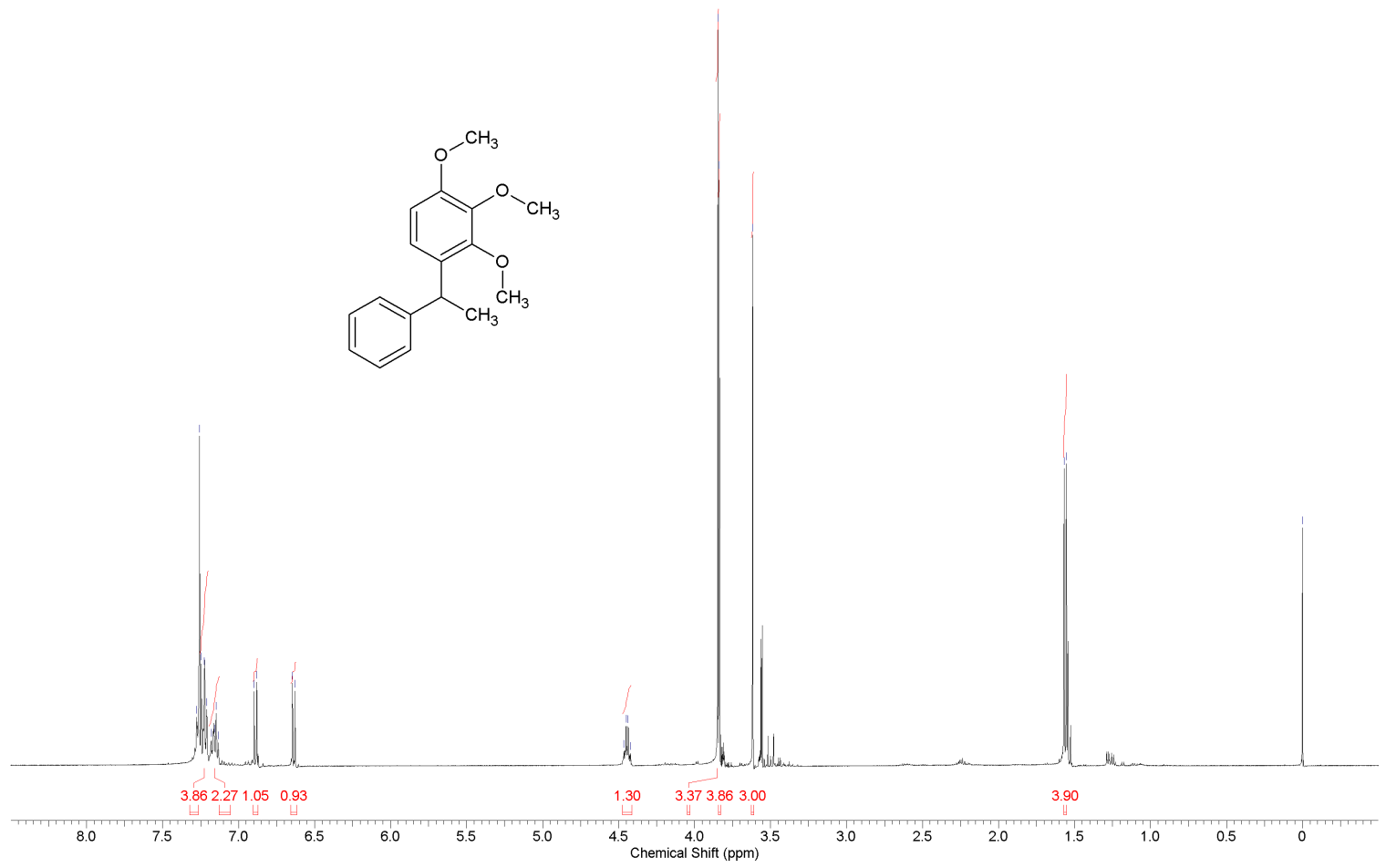

${ }^{1} \mathrm{H}$ NMR of 1,2-Dimethyl-4-(1-phenylethyl)-benzene (10)

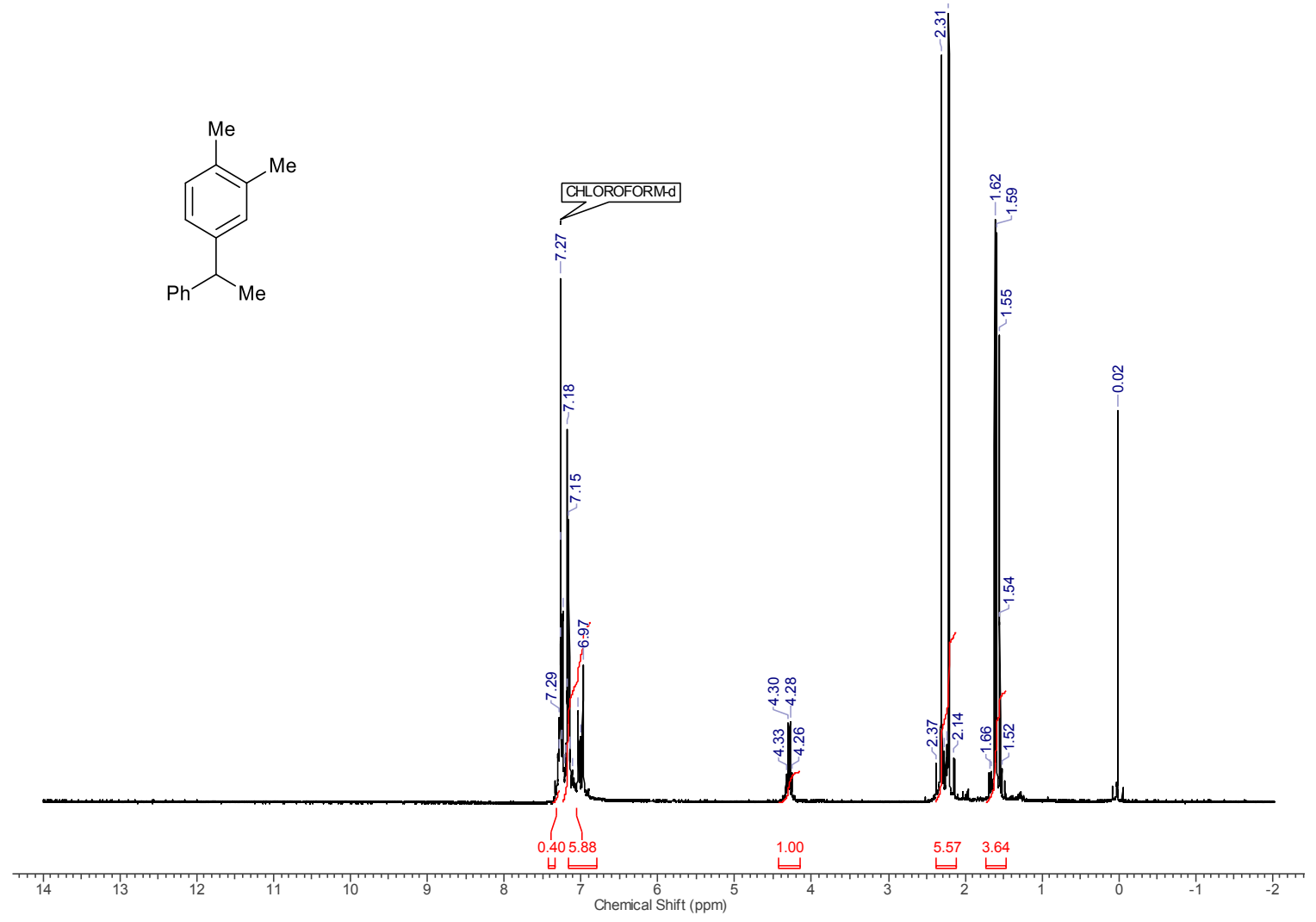


${ }^{1}$ H NMR of 1-Methyl-3-(1-phenylethyl)-1H-Indole (11) nmethylindole.e.sp<smiles>Cn1cc(C(c2ccccc2)c2ccccc2)c2ccccc21</smiles>

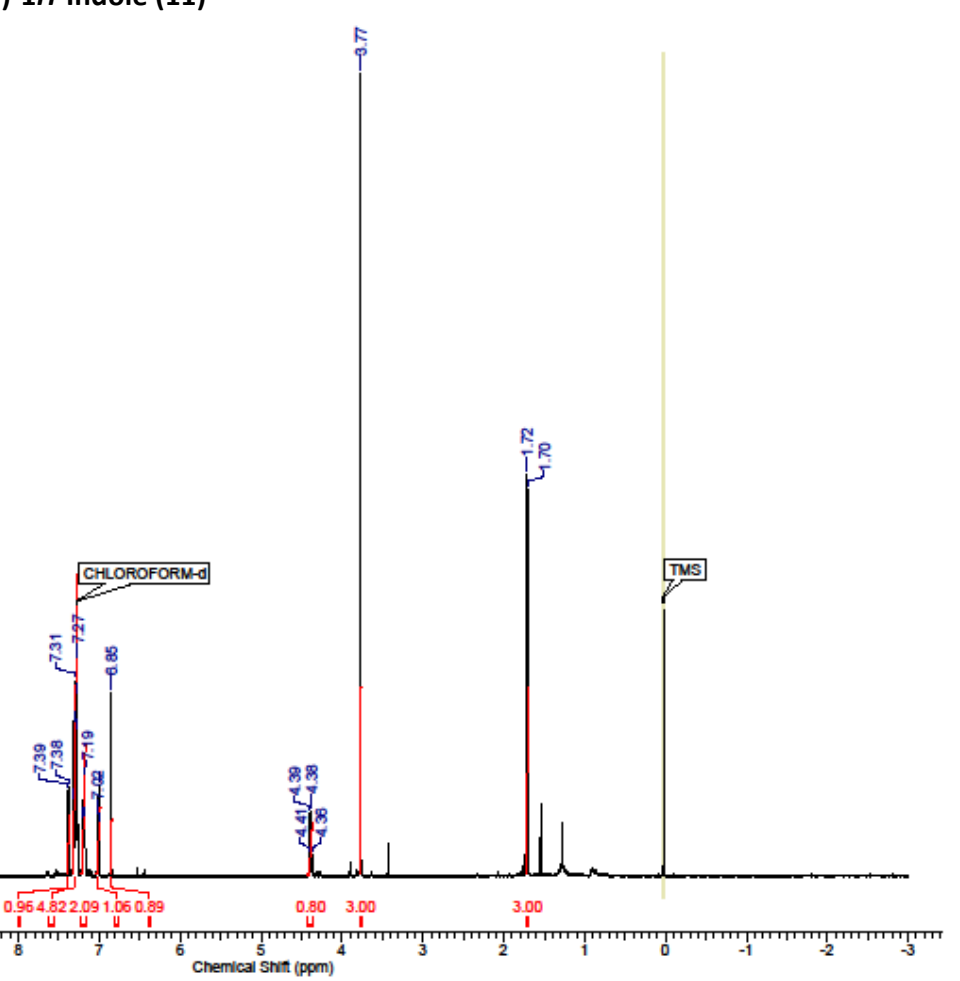

${ }^{1}$ H NMR of 1-Methoxy-4-[1-(4-methylphenyl)ethyl]benzene (12) 4methylstyrene.esp

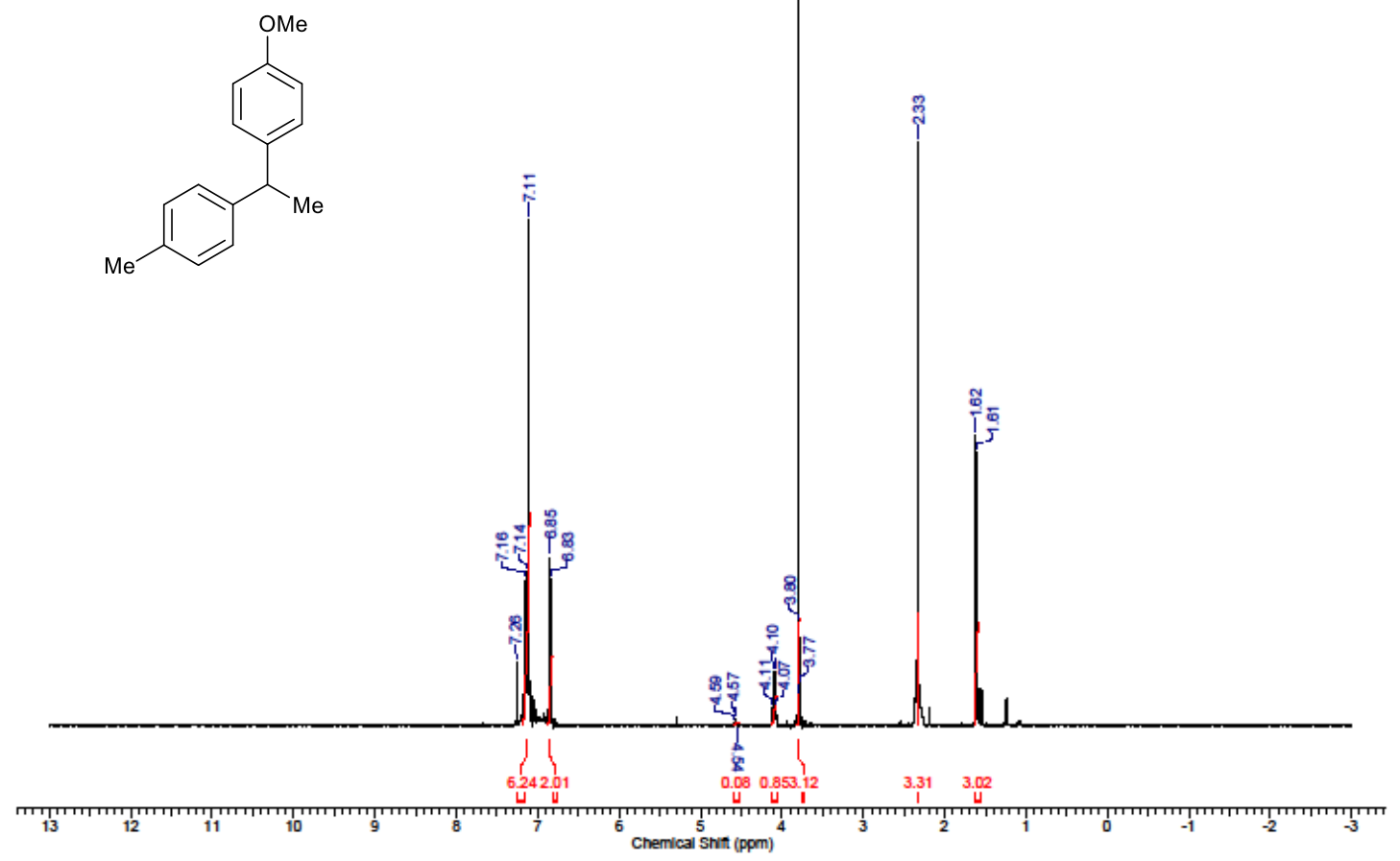


${ }^{1}$ H NMR of 1-Methoxy-4-[1-(4-fluorophenyl)ethyl]benzene (13a) and 1-methoxy-2-[1-(4fluorophenyl)ethyl]benzene (13b)

4nuorostyrene.esp<smiles>COc1ccc(C(C)c2ccc(F)cc2)cc1</smiles>

and<smiles>COc1ccccc1C(C)c1ccc(F)cc1</smiles>

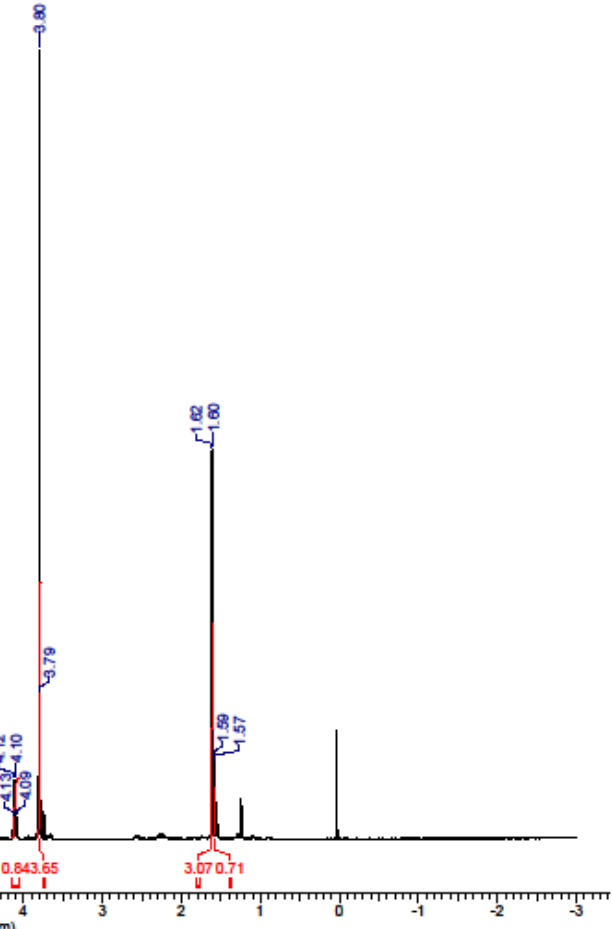

${ }^{1}$ H NMR of 1-Methoxy-4-(1-phenylpropyl)benzene (14a) and 1-methoxy-2-(1-phenylpropyl)benzene (14b) betamethylstrene.es<smiles>CCC(c1ccccc1)c1ccc(OC)cc1</smiles>

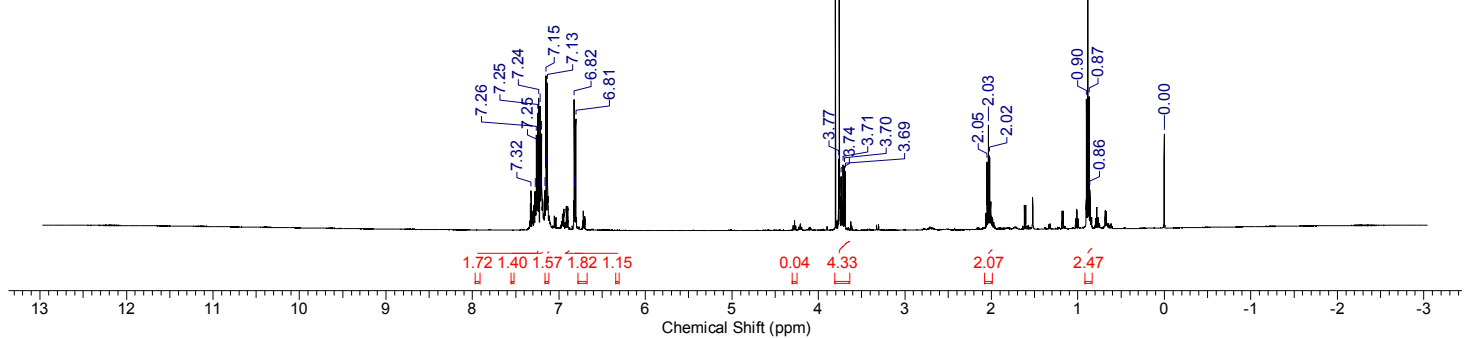


${ }^{1}$ H NMR of 1-(1,1-Diphenylethyl)-4-methoxybenzene (15)

alphenylethylene.esp<smiles>COc1ccc(C(C)(c2ccccc2)c2ccccc2)cc1</smiles>

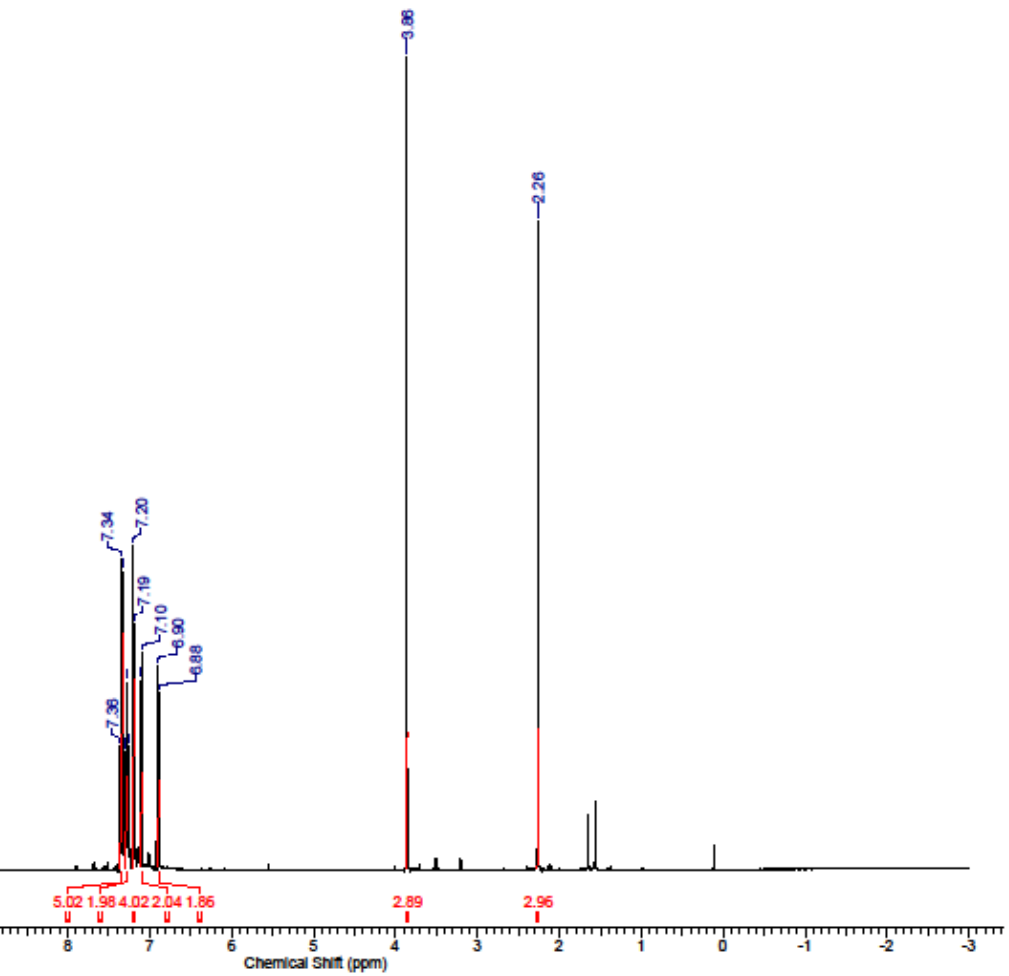

${ }^{1}$ H NMR of 1-(4-Methoxyphenyl)-2,3-dihydro-1H-indene (16a) and 1-(2-methoxyphenyl)-2,3-dihydro-1 $H$-indene (16b) Indene.esp<smiles>COc1ccc(C2CCc3ccccc32)cc1</smiles>

and<smiles>COc1ccccc1C1CCc2ccccc21</smiles>

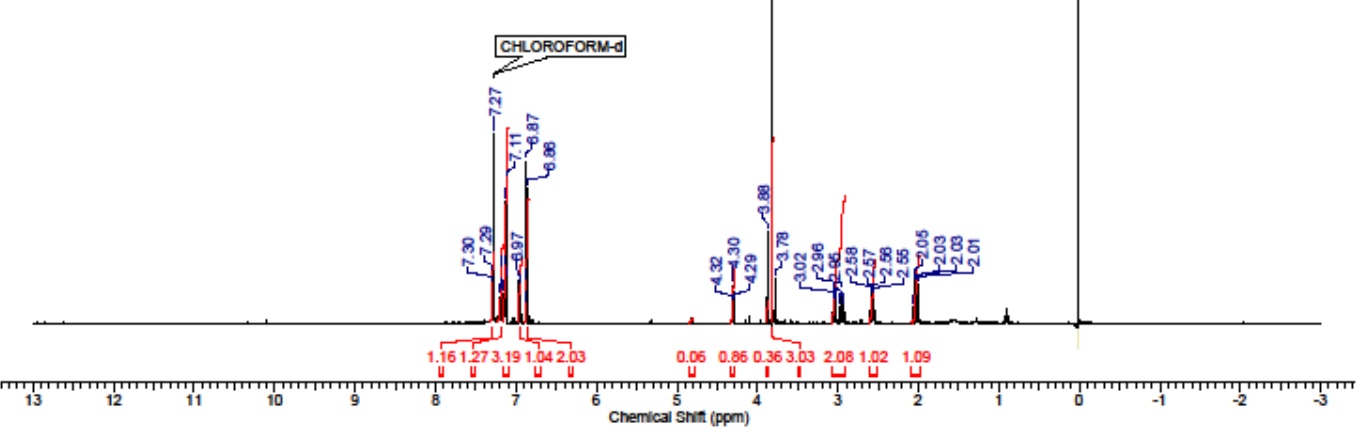


${ }^{1} \mathrm{H}$ NMR of 1-(4-Methoxyphenyl)-1,2,3,4-tetrahydronaphthalene (17a) and 1-(2-methoxyphenyl)-1,2,3,4tetrahydronaphthalene (17b)

DHN.esP<smiles>COc1ccc(C2CCCc3ccccc32)cc1</smiles>

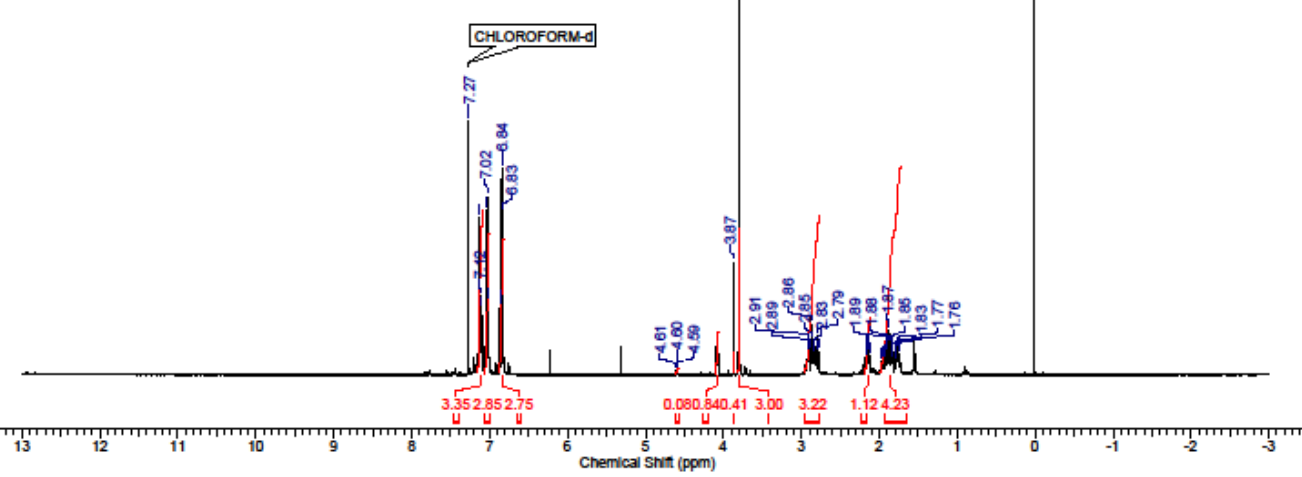

${ }^{1}$ H NMR of 1-Methoxy-4-(1-methylcyclohexyl)-benzene (18) methylcyclohexene.esp

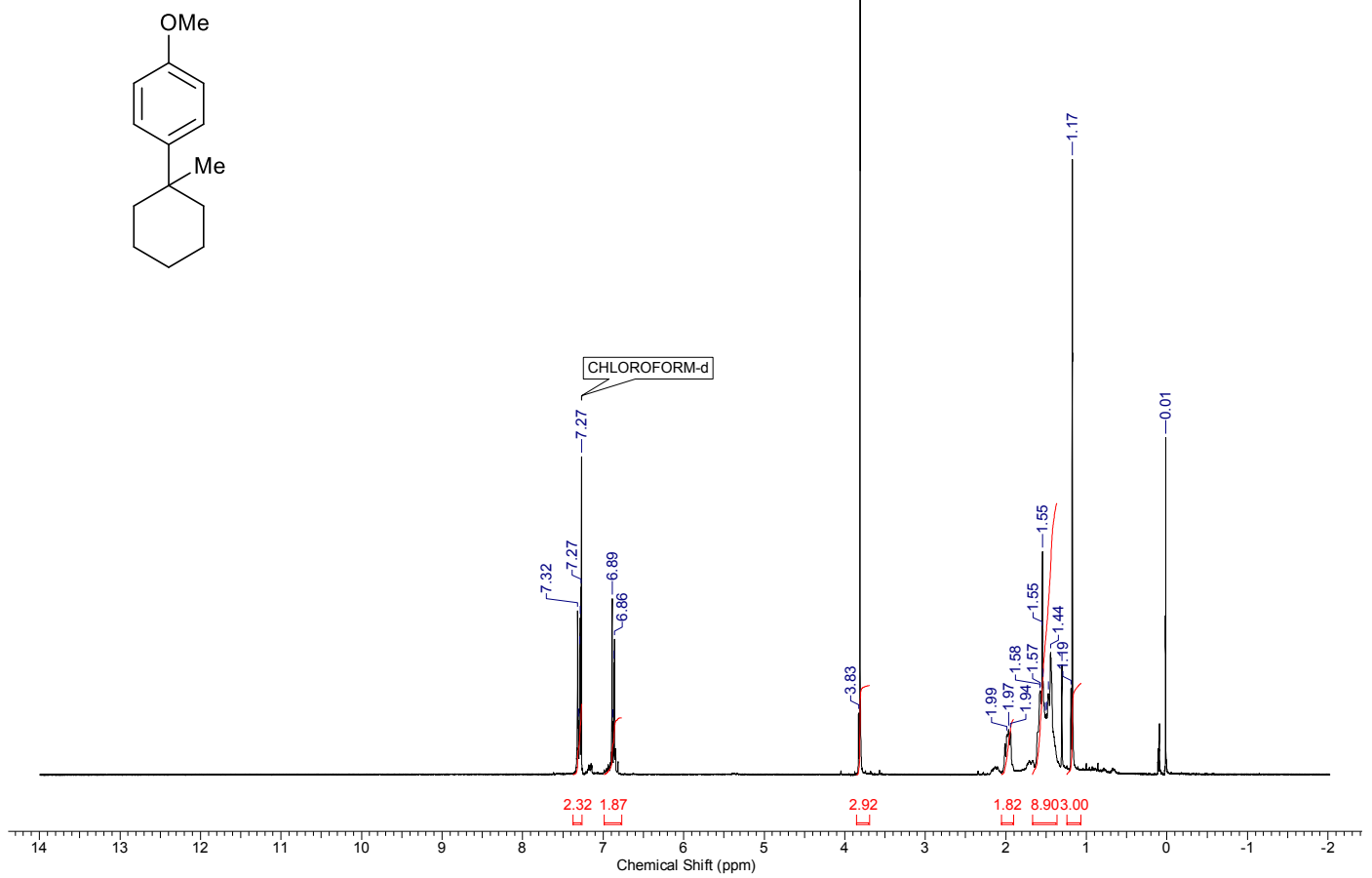


${ }^{1} \mathrm{H}$ NMR of 1-Cyclohexyl-2-methoxybenzene (20a) and 1-cyclohexyl-4-methoxybenzene (20b)

cyclohexene.esp and
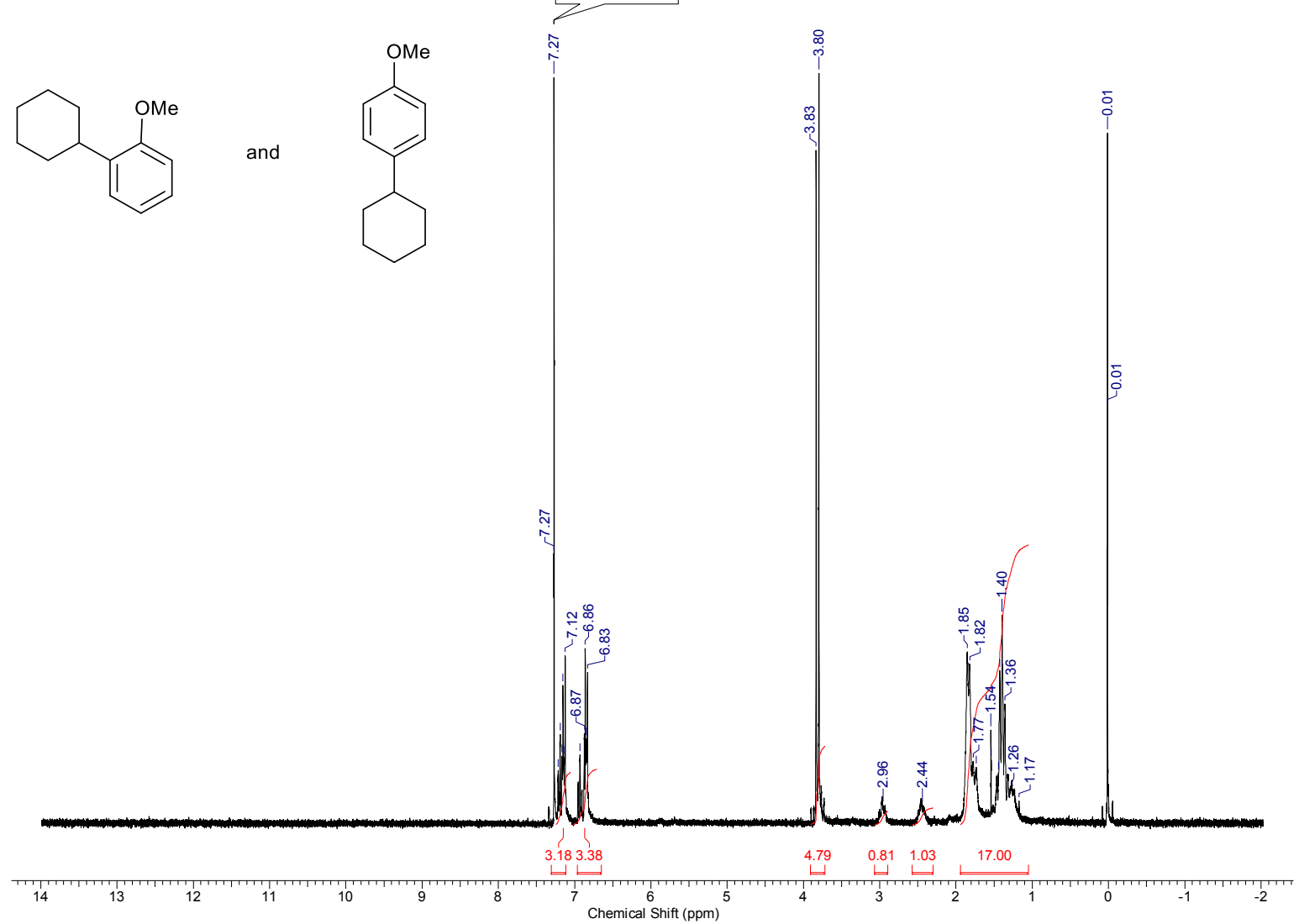

CHLOROFORM-d

$\overbrace{}^{\mathrm{CHLO} O \mathrm{O}}$
(1)

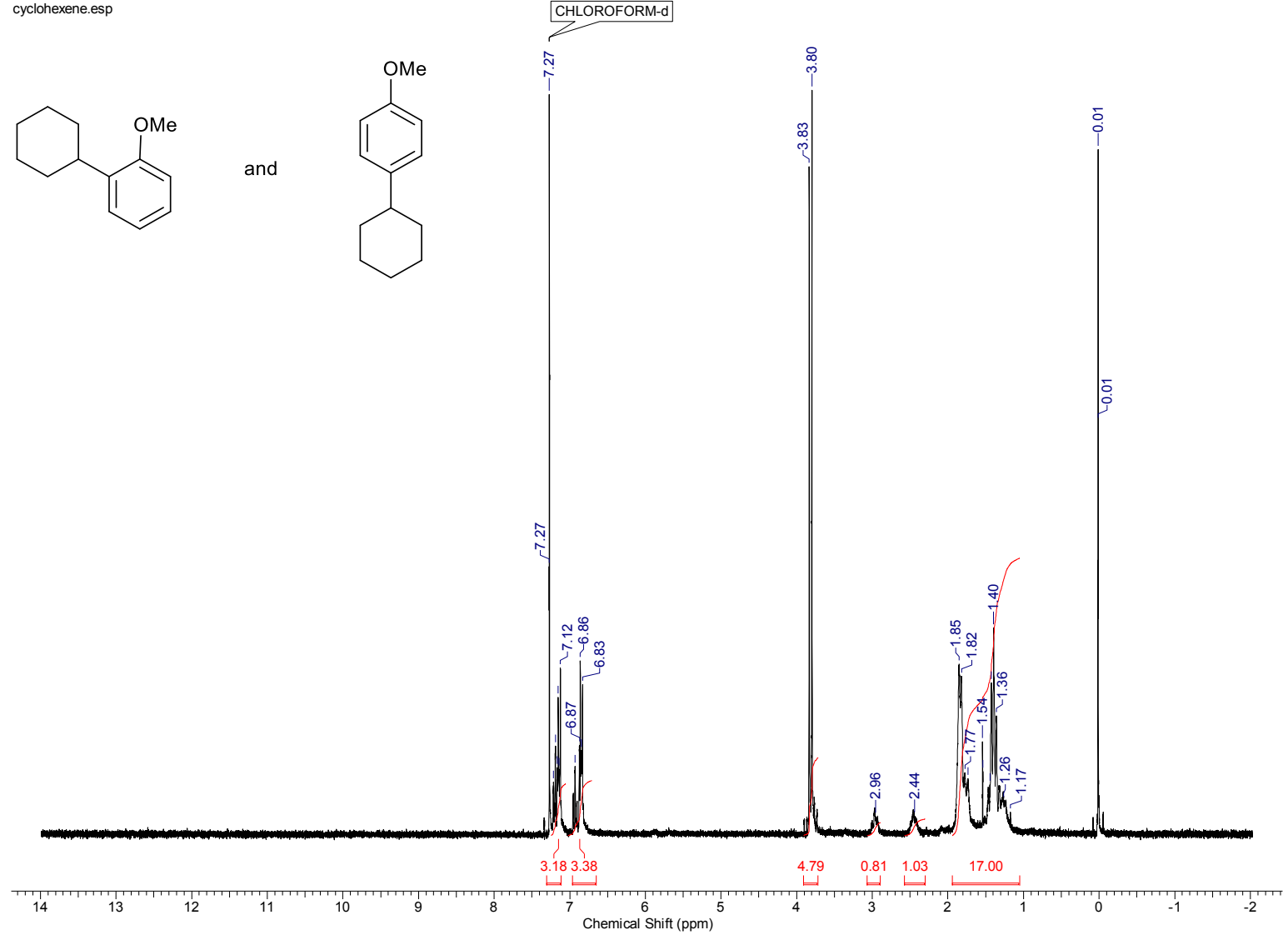

GC data for 1-Cyclopentyl-2-methoxybenzene (21a) and 1-cyclopentyl-4-methoxybenzene (21b)

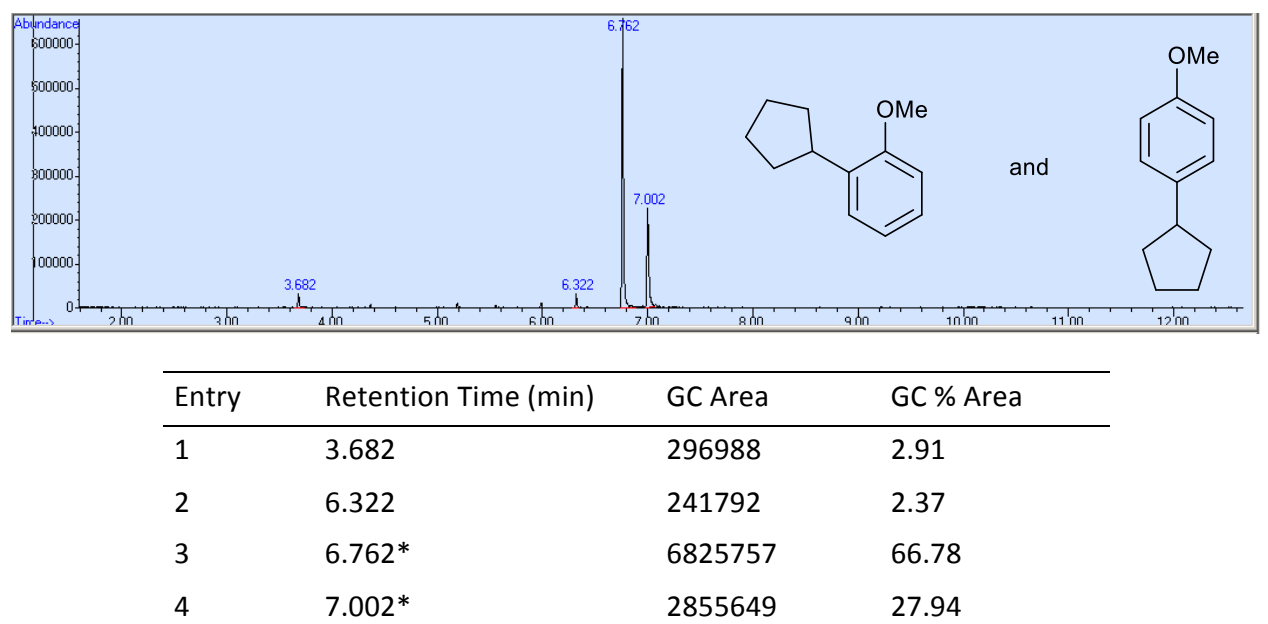

* denotes peaks that pertain to product. Note: Purity determined through GC area (uncorrected) after column chromatography. 